\title{
Graded Causation and Defaults*
}

\author{
Joseph Y. Halpern ${ }^{\dagger}$ \\ Cornell University \\ halpern@cs.cornell.edu
}

\author{
Christopher Hitchcock \\ California Institute of Technology \\ cricky@caltech.edu
}

September 6, 2013

\begin{abstract}
Recent work in psychology and experimental philosophy has shown that judgments of actual causation are often influenced by consideration of defaults, typicality, and normality. A number of philosophers and computer scientists have also suggested that an appeal to such factors can help deal with problems facing existing accounts of actual causation. This paper develops a flexible formal framework for incorporating defaults, typicality, and normality into an account of actual causation. The resulting account takes actual causation to be both graded and comparative. We then show how our account would handle a number of standard cases.
\end{abstract}

\section{Introduction}

The notion of actual causation (also called "singular causation", "token causation", or just "causation" in the literature), the relation that we judge to hold when we affirm a statement that one particular event caused another, is ubiquitous in descriptions of the world. For example, the following claims all describe relations of actual causation:

- Paula's putting poison in the tea caused Victoria to die.

- A lightning strike caused the forest fire.

${ }^{*}$ For helpful comments and discussion, we would like to thank Luke Glynn, Franz Huber, Josh Knobe, Jonathan Livengood, Laurie Paul, Jim Woodward, two anonymous referees, members of the McDonnell Causal Learning Collaborative, and audience members at the Association of Symbolic Logic meeting (Chicago 2010), the Society for Exact Philosophy (Kansas City 2010), the Formal Epistemology Festival (Toronto 2010), the Workshop on Actual Causation (Konstanz 2010), Ohio University, California Institute of Technology, Rutgers University, and the University of California at San Diego.

${ }^{\dagger}$ Supported in part by NSF grants IIS-0812045 and IIS-0911036, AFOSR grants FA9550-08-1-0438 and FA9550-05-1-0055, and ARO grant W911NF-09-1-0281. 
- The ignition of the rum aboard the ship caused Lloyd's of London to suffer a large financial loss.

Not surprisingly, this relation has been the subject of many analyses, including Lewis's classic paper "Causation" [Lewis 1973]. Actual causation has been of particular interest in philosophy and the law, in part because of its connection with issues of moral and legal responsibility (see for example, Moore [2009] for a detailed discussion of these connections).

Getting an adequate definition of actual causation has proved exceedingly difficult. There are literally hundreds of papers in fields as diverse as philosophy, law, economics, physics, and computer science proposing and criticizing definitions of actual causation. Recent attempts to provide an analysis based on structural equations [Glymour and Wimberly 2007; Hall 2007; Halpern and Pearl 2001; Hitchcock 2001; Pearl 2000; Woodward 2003] have met with some success. But these accounts also seem to have a significant problem: The definitions of causality based on structural equations all appeal to counterfactuals. Hume [1748] proposed (perhaps inadvertently) that $A$ is a cause of $B$ if, had $A$ not happened, $B$ would not have happened. As is well known, this definition is too naive. To take an example due to Wright [1985], suppose that Victoria, the victim, drinks a cup of tea poisoned by Paula, but before the poison takes effect, Sharon shoots Victoria, and she dies. We would like to call Sharon's shot the cause of Victoria's death, but if Sharon hadn't shot, Victoria would have died in any case. This is typically dealt with by considering the contingency where Paula does not administer the poison. Under that contingency, Victoria dies if Sharon shoots, and otherwise does not. To prevent the poisoning from also being a cause of Paula's death, constraints are placed on the contingencies that can be considered; the different approaches differ in the details regarding these constraints.

Any definition of causation that appeals to counterfactuals will face problems in cases where there are isomorphic patterns of counterfactual dependence, but different causal judgments seem appropriate. Consider, for example, cases of causation by omission. Suppose that while a homeowner is on vacation, the weather is hot and dry, her next door neighbor does not water her flowers, and the flowers die. Had the weather been different, or had her next door neighbor watered the flowers, they would not have died. The death of the flowers depends counterfactually upon both of these factors. So it would seem that a theory of causation based upon counterfactuals cannot discriminate between these factors. Yet several authors, including Beebee [2004] and Moore [2009], have argued that the weather is a cause of the flowers' death, while the neighbor's negligence is not. Let us call this the problem of isomorphism.

In fact, this case points to an even deeper problem. There is actually a range of different opinions in the literature about whether to count the neighbor's negligence as an actual cause of the flowers' death. (See Section 7.1 for details and references). Prima facie, it does not seem that any theory of actual causation can respect all of these judgments without lapsing into inconsistency. Let us call this the problem of disagreement.

One approach to solving these problems that has been gaining increasing popularity (see, e.g., [Hall 2007; Halpern 2008; Hitchcock 2007; Menzies 2004; Menzies 2007]) is to incorporate defaults, typicality, and normality into an account of actual causation. These approaches 
gain further support from empirical results showing that such considerations do in fact influence people's judgments about actual causation (see e.g. Cushman et al. [2008], Hitchcock and Knobe [2009], and Knobe and Fraser [2008].)

In the present paper, we develop this approach in greater detail. We represent these factors using a ranking on "possible worlds" that we call a normality ordering. Our formalism is intended to be flexible. One can use a normality ordering to represent many different kinds of considerations that might affect judgments of actual causation. We leave it open for philosophers and psychologists to continue debating about what kinds of factors $d o$ affect judgments of actual causation, and what kinds of factors should affect judgments of causation. Our goal is not to settle these issues here. Rather, our intent is to provide a flexible formal framework for representing a variety of different kinds of causal judgment.

Our approach allows us to deal with both the problem of isomorphism and the problem of disagreement. It can deal with the problem of isomorphism, since cases that have isomorphic structures of counterfactual dependence can have non-isomorphic normality orderings. It can deal with the problem of disagreement, since people can disagree about claims of actual causation despite being in agreement about the underlying structure of a particular case because they are using different normality orderings.

The approach has some additional advantages. Specifically, it allows us to move away from causality being an all or nothing assignment-either $A$ is a cause of $B$ or it is not-to a more "graded" notion of causality. We can then talk about one event being viewed as more of a cause than another. To the extent that we tend to view one event as "the" cause, it is because it is the one that is the "best" cause. 1

For definiteness, we start with the definition of causality given by Halpern and Pearl [2005] (HP from now on), and add normality to that. However, it is not our intention in this paper to argue for the superiority of the HP definition over others, nor to argue that our revision of the HP account yields a fully adequate definition? Indeed, our recipe for modifying the HP definition can be applied to many other accounts, and the resulting treatment of the various cases will be similar 3

The rest of the paper is organized as follows. In the next two sections, we review the causal modeling framework that we employ, and the HP definition of actual causation. Readers who are already familiar with these may skim these sections. In Section 4 , we briefly review some of the problems faced by the HP theory. In Section 5, we informally introduce the notions of defaults, typicality, and normality, and provide some further motivation for incorporating these notions into a theory of actual causation. Section 6 contains our formal treatment of these notions, and presents our revised, graded definition of actual causation. We conclude by

\footnotetext{
${ }^{1}$ Chockler and Halpern [2004] introduce a notion of responsibility that also can be viewed as providing a more graded notion of causality, but it is quite different in spirit from that considered here.

${ }^{2}$ In particular, our current proposal does not address putative counterexamples the the HP definition raised by Weslake [2011], nor the ones involving voting scenarios described by Glymour et al. [2010] and Livengood [2013]. We hope to address these examples in future work.

${ }^{3}$ One likely exception is the treatment of legal causation in Section 7.6. Here the treatment does depend upon the the details of HP definition.
} 
applying the revised definition to a number of examples.

\section{Causal Models}

The HP approach models the world using random variables and their values. For example, if we are trying to determine whether a forest fire was caused by lightning or an arsonist, we can construct a model using three random variables:

- $F F$ for forest fire, where $F F=1$ if there is a forest fire and $F F=0$ otherwise;

- $L$ for lightning, where $L=1$ if lightning occurred and $L=0$ otherwise;

- $M$ for match (dropped by arsonist), where $M=1$ if the arsonist drops a lit match, and $M=0$ otherwise.

The choice of random variables determines the language used to frame the situation. Although there is no "right" choice, clearly some choices are more appropriate than others. For example, when trying to determine the cause of the forest fire, if there is no random variable corresponding to the lightning in a model then, in that model, we cannot hope to conclude that lightning is a cause of the forest fire.

Some random variables may have a causal influence on others. This influence is modeled by a set of structural equations. For example, to model the fact that if a match is lit or lightning strikes then a fire starts, we could use the random variables $M, F F$, and $L$ as above, with the equation

$$
F F=\max (L, M)
$$

Since the value of $F F$ is the maximum of the values of $L$ and $M, F F$ is 1 if either of $L$ and $M$ is 1 . Alternatively, if a fire requires both a lightning strike and a dropped match (perhaps the wood is so wet that it needs two sources of fire to get going), the appropriate equation for $F F$ would be

$$
F F=\min (L, M)
$$

the value of $F F$ is the minimum of the values of $L$ and $M$. The only way that $F F=1$ is if both $L=1$ and $M=1$. For future reference, we call the model that uses the first equation the disjunctive model, and the one that uses the second equation the conjunctive model.

The equality signs in these equations should be thought of more like assignment statements in programming languages than normal algebraic equalities. For example, the first equation tells us that once we set the values of $M$ and $L$, then the value of $F F$ is set to their maximum. This relation is not symmetric; if a forest fire starts some other way, that does not force the value of either $M$ or $L$ to be 1 . This asymmetry corresponds to the asymmetry in what Lewis 
[1979] calls "non-backtracking" counterfactuals. Suppose that there actually was no lightning, and the arsonist did not drop a match. Then (using non-backtracking counterfactuals), we would say that if lightning had struck or the arsonist had lit her match, then there would have been a fire. However, we would not say that if there had been a fire, then either lightning would have struck, or the arsonist would have lit her match.

These models are somewhat simplistic. Lightning does not always result in a fire, nor does dropping a lit match. One way of dealing with this would be to make the assignment statements probabilistic. For example, we could say that the probability that $F F=1$ conditional on $L=1$ is .8. This approach would lead to rather complicated definitions. Another approach would be to use enough variables to capture all the conditions that determine whether there is a forest fire. For example, we could add variables that talk about the dryness of the wood, the amount of undergrowth, the presence of sufficient oxygen, and so on. If a modeler does not want to add all these variables explicitly (the details may simply not be relevant to the analysis), another alternative is to use a single variable, say $W$, that intuitively incorporates all the relevant factors, without describing them explicitly. Thus, $W$ could take the value 1 if conditions are such that a lightning strike or a dropped match would suffice to start a fire, and 0 otherwise. The final possibility, which we will adopt, is to take these factors to be "built in" to the equation $F F=\min (L, M)$. That is, in using this equation, we are not claiming that a dropped match or a lightning strike will always cause a fire, but only that the actual conditions are such either of these would in fact start a fire.

It is also clear that we could add further variables to represent the causes of $L$ and $M$ (and the causes of those causes, and so on). We instead represent these causes with a single variable $U$. The value of $U$ determines whether the lightning strikes and whether the match is dropped by the arsonist. In this way of modeling things, $U$ takes on four possible values of the form $(i, j)$, where $i$ and $j$ are both either 0 or 1 . Intuitively, $i$ describes whether the external conditions are such that the lightning strikes (and encapsulates all the conditions, such as humidity and temperature, that affect whether the lightning strikes); and $j$ describes whether the external conditions are such that the arsonist drops the match (and thus encapsulates the psychological conditions that determine whether the arsonist drops the match).

It is conceptually useful to split the random variables into two sets: the exogenous variables, whose values are determined by factors outside the model, and the endogenous variables, whose values are ultimately determined by the exogenous variables. In the forest-fire example, the variables $M, L$, and $F F$ are endogenous. However, we do not want to concern ourselves with the factors that make the arsonist drop the match or the factors that cause lightning. Thus we do not include endogenous variables for these factors, but rather incorporate them into the exogenous variable(s).

Formally, a causal model $M$ is a pair $(\mathcal{S}, \mathcal{F})$, where $\mathcal{S}$ is a signature, which explicitly lists the endogenous and exogenous variables and characterizes their possible values, and $\mathcal{F}$ defines a set of modifiable structural equations, relating the values of the variables. A signature $\mathcal{S}$ is a tuple $(\mathcal{U}, \mathcal{V}, \mathcal{R})$, where $\mathcal{U}$ is a set of exogenous variables, $\mathcal{V}$ is a set of endogenous variables, and $\mathcal{R}$ associates with every variable $Y \in \mathcal{U} \cup \mathcal{V}$ a nonempty set $\mathcal{R}(Y)$ of possible values for $Y$ 
(that is, the set of values over which $Y$ ranges). As suggested above, in the forest-fire example, we have $\mathcal{U}=\{U\}$, where $U$ is the exogenous variable, $\mathcal{R}(U)$ consists of the four possible values of $U$ discussed earlier, $\mathcal{V}=\{F F, L, M\}$, and $\mathcal{R}(F F)=\mathcal{R}(L)=\mathcal{R}(M)=\{0,1\}$.

$\mathcal{F}$ associates with each endogenous variable $X \in \mathcal{V}$ a function denoted $F_{X}$ such that

$$
F_{X}:\left(\times_{U \in \mathcal{U}} \mathcal{R}(U)\right) \times\left(\times_{Y \in \mathcal{V}-\{X\}} \mathcal{R}(Y)\right) \rightarrow \mathcal{R}(X) .
$$

This mathematical notation just makes precise the fact that $F_{X}$ determines the value of $X$, given the values of all the other variables in $\mathcal{U} \cup \mathcal{V}$. If there is one exogenous variable $U$ and three endogenous variables, $X, Y$, and $Z$, then $F_{X}$ defines the values of $X$ in terms of the values of $Y, Z$, and $U$. For example, we might have $F_{X}(u, y, z)=u+y$, which is usually written as $X=U+Y 4$ Thus, if $Y=3$ and $U=2$, then $X=5$, regardless of how $Z$ is set.

In the running forest-fire example, where $U$ has four possible values of the form $(i, j)$, the $i$ value determines the value of $L$ and the $j$ value determines the value of $M$. Although $F_{L}$ gets as arguments the values of $U, M$, and $F F$, in fact, it depends only on the (first component of) the value of $U$; that is, $F_{L}((i, j), m, f)=i$. Similarly, $F_{M}((i, j), l, f)=j$. In this model, the value of $F F$ depends only on the value of $L$ and $M$. How it depends on them depends on whether we are considering the conjunctive model or the disjunctive model.

It is sometimes helpful to represent a causal model graphically. Each node in the graph corresponds to one variable in the model. An arrow from one node, say $L$, to another, say $F F$, indicates that the former variable figures as a nontrivial argument in the equation for the latter. Thus, we could represent either the conjunctive or the disjunctive model using Figure 1(a). Often we omit the exogenous variables from the graph; in this case, we would represent either model using Figure 1(b). Note that the graph conveys only the qualitative pattern of dependence; it does not tell us how one variable depends on others. Thus the graph alone does not allow us to distinguish between the disjunctive and the conjunctive models.

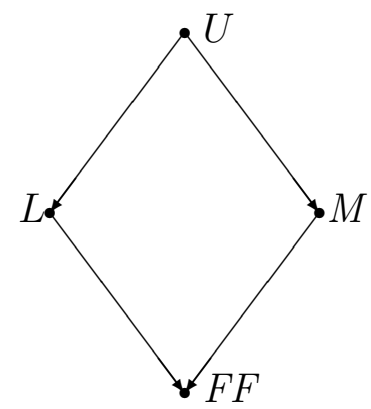

(a)

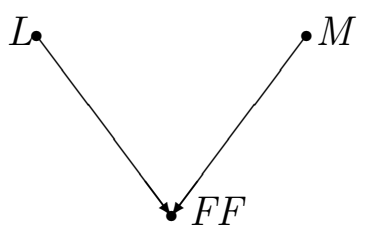

(b)

Figure 1: A graphical representation of structural equations.

\footnotetext{
${ }^{4}$ Again, the fact that $X$ is assigned $U+Y$ (i.e., the value of $X$ is the sum of the values of $U$ and $Y$ ) does not imply that $Y$ is assigned $X-U$; that is, $F_{Y}(U, X, Z)=X-U$ does not necessarily hold.
} 
The key role of the structural equations is to define what happens in the presence of external interventions. For example, we can explain what would happen if one were to intervene to prevent the arsonist from dropping the match. In the disjunctive model, there is a forest fire exactly exactly if there is lightning; in the conjunctive model, there is definitely no fire. Setting the value of some variable $X$ to $x$ in a causal model $M=(\mathcal{S}, \mathcal{F})$ by means of an intervention results in a new causal model denoted $M_{X=x} . M_{X=x}$ is identical to $M$, except that the equation for $X$ in $\mathcal{F}$ is replaced by $X=x$. We sometimes talk of "fixing" the value of $X$ at $x$, or "setting" the value of $X$ to $x$. These expressions should also be understood as referring to interventions on the value of $X$. Note that an "intervention" does not necessarily imply human agency. The idea is rather that some independent process overrides the existing causal structure to determine the value of one or more variables, regardless of the value of its (or their) usual causes. Woodward [2003] gives a detailed account of such interventions. Lewis [1979] suggests that we think of the antecedents of non-backtracking counterfactuals as being made true by "small miracles". These "small miracles" would also count as interventions.

It may seem circular to use causal models, which clearly already encode causal information, to define actual causation. Nevertheless, there is no circularity. The models do not directly represent relations of actual causation. Rather, they encode information about what would happen under various possible interventions. Equivalently, they encode information about which non-backtracking counterfactuals are true. We will say that the causal models represent (perhaps imperfectly or incompletely) the underlying causal structure. While there may be some freedom of choice regarding which variables are included in a model, once an appropriate set of variables has been selected, there should be an objective fact about which equations among those variables correctly describe the effects of interventions on some particular system of interest. 5

There are (at least) two ways of thinking about the relationship between the equations of a causal model and the corresponding counterfactuals. One way, suggested by Hall [2007], is to attempt to analyze the counterfactuals in non-causal terms, perhaps along the lines of [Lewis 1979]. The equations of a causal model would then be formal representations of these counterfactuals. A second approach, favored by Pearl [2000], is to understand the equations as representations of primitive causal mechanisms. These mechanisms then ground the counterfactuals. While we are more sympathetic to the second approach, nothing in the present paper depends on this. In either case, the patterns of dependence represented by the equations are distinct from relations of actual causation.

In a causal model, it is possible that the value of $X$ can depend on the value of $Y$ (that is, the equation $F_{X}$ is such that changes in $Y$ can change the value of $X$ ) and the value of $Y$ can depend on the value of $X$. Intuitively, this says that $X$ can potentially affect $Y$ and that $Y$ can potentially affect $X$. While allowed by the framework, this type of situation does not happen in the examples of interest; dealing with it would complicate the exposition. Thus, for ease of exposition, we restrict attention here to what are called recursive (or acyclic) models. This is

\footnotetext{
${ }^{5}$ Although as Halpern and Hitchcock [2010] note, some choices of variables do not give rise to well-defined equations. This would count against using that set of variables to model the system of interest.
} 
the special case where there is some total ordering $<$ of the endogenous variables (the ones in $\mathcal{V})$ such that if $X<Y$, then $X$ is independent of $Y$, that is, $F_{X}(\ldots, y, \ldots)=F_{X}\left(\ldots, y^{\prime}, \ldots\right)$ for all $y, y^{\prime} \in \mathcal{R}(Y)$. If $X<Y$, then the value of $X$ may affect the value of $Y$, but the value of $Y$ cannot affect the value of $X$. Intuitively, if a theory is recursive, there is no feedback. The graph representing an acyclic causal model does not contain any directed paths leading from a variable back into itself, where a directed path is a sequence of arrows aligned tip to tail.

If $M$ is an acyclic causal model, then given a context, that is, a setting $\vec{u}$ for the exogenous variables in $\mathcal{U}$, there is a unique solution for all the equations. We simply solve for the variables in the order given by $<$. The value of the variables that come first in the order, that is, the variables $X$ such that there is no variable $Y$ such that $Y<X$, depend only on the exogenous variables, so their value is immediately determined by the values of the exogenous variables. The values of variables later in the order can be determined once we have determined the values of all the variables earlier in the order.

There are many nontrivial decisions to be made when choosing the structural model to describe a given situation. One significant decision is the set of variables used. As we shall see, the events that can be causes and those that can be caused are expressed in terms of these variables, as are all the intermediate events. The choice of variables essentially determines the "language" of the discussion; new events cannot be created on the fly, so to speak. In our running example, the fact that there is no variable for unattended campfires means that the model does not allow us to consider unattended campfires as a cause of the forest fire.

It is not always straightforward to decide what the "right" causal model is in a given situation. In particular, there may be disagreement about which variables to use. If the variables are well-chosen, however, it should at least be clear what the equations relating them should be, if the behavior of the system is understood. These decisions often lie at the heart of determining actual causation in the real world. While the formalism presented here does not provide techniques to settle disputes about which causal model is the right one, at least it provides tools for carefully describing the differences between causal models, so that it should lead to more informed and principled decisions about those choices. (Again, see [Halpern and Hitchcock 2010] for further discussion of these issues.)

\section{The HP Definition of Actual Causation}

To formulate the HP definition of actual cause, it is helpful to have a formal language for counterfactuals and interventions. Given a signature $\mathcal{S}=(\mathcal{U}, \mathcal{V}, \mathcal{R})$, a primitive event is a formula of the form $X=x$, for $X \in \mathcal{V}$ and $x \in \mathcal{R}(X)$. A causal formula (over $\mathcal{S}$ ) is one of the form $\left[Y_{1}=y_{1}, \ldots, Y_{k}=y_{k}\right] \varphi$, where

- $\varphi$ is a Boolean combination of primitive events,

- $Y_{1}, \ldots, Y_{k}$ are distinct variables in $\mathcal{V}$, and

- $y_{i} \in \mathcal{R}\left(Y_{i}\right)$. 
Such a formula is abbreviated $[\vec{Y}=\vec{y}] \varphi$. The special case where $k=0$ is abbreviated as $\varphi$. Intuitively, $\left[Y_{1}=y_{1}, \ldots, Y_{k}=y_{k}\right] \varphi$ says that $\varphi$ would hold if each $Y_{i}$ were set to $y_{i}$ by an intervention, for $i=1, \ldots, k$.

A causal formula $\varphi$ is true or false in a causal model, given a context. We write $(M, \vec{u}) \models \varphi$ if the causal formula $\varphi$ is true in causal model $M$ given context $\vec{u}$. The $\models$ relation is defined inductively. $(M, \vec{u}) \models X=x$ if the variable $X$ has value $x$ in the unique solution to the equations in $M$ in context $\vec{u}$ (that is, the unique vector of values for the endogenous variables that simultaneously satisfies all equations in $M$ with the variables in $\mathcal{U}$ set to $\vec{u}$ ). The truth of conjunctions and negations is defined in the standard way. Finally, $(M, \vec{u}) \models[\vec{Y}=\vec{y}] \varphi$ if $\left(M_{\vec{Y}=\vec{y}}, \vec{u}\right) \models \varphi$.

For example, if $M$ is the disjunctive causal model for the forest fire, and $u$ is the context where there is lightning and the arsonist drops the lit match, then $(M, u) \models[M=0](F F=1)$, since even if the arsonist is somehow prevented from dropping the match, the forest burns (thanks to the lightning); similarly, $(M, u) \models[L=0](F F=1)$. However, $(M, u) \models[L=$ $0 ; M=0](F F=0)$ : if the arsonist does not drop the lit match and the lightning does not strike, then the forest does not burn.

The HP definition of causality, like many others, is based on counterfactuals. The idea is that $A$ is a cause of $B$ if, if $A$ hadn't occurred (although it did), then $B$ would not have occurred. This idea goes back to at least Hume [1748, Section VIII], who said:

We may define a cause to be an object followed by another, ..., if the first object had not been, the second never had existed.

This is essentially the but-for test, perhaps the most widely used test of actual causation in tort adjudication. The but-for test states that an act is a cause of injury if and only if, but for the act (i.e., had the the act not occurred), the injury would not have occurred. David Lewis [1973] has also advocated a counterfactual definition of causation.

There are two well-known problems with this definition. The first can be seen by considering the disjunctive causal model for the forest fire again. Suppose that the arsonist drops a match and lightning strikes. Which is the cause? According to a naive interpretation of the counterfactual definition, neither is. If the match hadn't dropped, then the lightning would still have struck, so there would have been a forest fire anyway. Similarly, if the lightning had not occurred, there still would have been a forest fire. As we shall see, the HP definition declares both lightning and the arsonist actual causes of the fire.

A more subtle problem is that of preemption, where there are two potential causes of an event, one of which preempts the other. Preemption is illustrated by the following story, due to Hitchcock [2007]:

An assassin puts poison in a victim's drink. If he hadn't poisoned the drink, a backup assassin would have. The victim drinks the poison and dies.

Common sense suggests that the assassin's poisoning the drink caused the victim to die. However, it does not satisfy the naive counterfactual definition either; if the assassin hadn't poisoned the drink, the backup would have, and the victim would have died anyway. 
The HP definition deals with these problems by defining actual causation as counterfactual dependence under certain contingencies. In the forest-fire example, the forest fire does counterfactually depend on the lightning under the contingency that the arsonist does not drop the match; similarly, the forest fire depends counterfactually on the arsonist's match under the contingency that the lightning does not strike. In the poisoning example, the victim's death does counterfactually depend on the first assassin's poisoning the drink under the contingency that the backup does not poison the drink (perhaps because she is not present). However, we need to be careful here to limit the contingencies that can be considered. We do not want to count the backup assassin's presence as an actual cause of death by considering the contingency where the first assassin does not poison the drink. We consider the first assassin's action to be the cause of death because it was her poison that the victim consumed. Somehow the definition must capture this obvious intuition. A big part of the challenge of providing an adequate definition of actual causation comes from trying get these restrictions just right.

With this background, we now give the HP definition of actual causation 6 The definition is relative to a causal model (and a context); $A$ may be a cause of $B$ in one causal model but not in another. The definition consists of three clauses. The first and third are quite simple; all the work is going on in the second clause.

The types of events that the HP definition allows as actual causes are ones of the form $X_{1}=x_{1} \wedge \ldots \wedge X_{k}=x_{k}$-that is, conjunctions of primitive events; this is often abbreviated as $\vec{X}=\vec{x}$. The events that can be caused are arbitrary Boolean combinations of primitive events. The definition does not allow statements of the form " $A$ or $A^{\prime}$ is a cause of $B$ ", although this could be treated as being equivalent to "either $A$ is a cause of $B$ or $A^{\prime}$ is a cause of $B$ ". On the other hand, statements such as " $A$ is a cause of $B$ or $B^{\prime \prime}$ " are allowed; as we shall see, this is not equivalent to "either $A$ is a cause of $B$ or $A$ is a cause of $B^{\prime \prime}$.

Definition 3.1: (Actual cause) [Halpern and Pearl 2005] $\vec{X}=\vec{x}$ is an actual cause of $\varphi$ in $(M, \vec{u})$ if the following three conditions hold:

AC1. $(M, \vec{u}) \models(\vec{X}=\vec{x})$ and $(M, \vec{u}) \models \varphi$.

AC2. There is a partition of $\mathcal{V}$ (the set of endogenous variables) into two subsets $\vec{Z}$ and $\vec{W}$ with $\vec{X} \subseteq \vec{Z}$, and settings $\vec{x}^{\prime}$ and $\vec{w}$ of the variables in $\vec{X}$ and $\vec{W}$, respectively, such that if $(M, \vec{u}) \models Z=z^{*}$ for all $Z \in \vec{Z}$, then both of the following conditions hold:

(a) $(M, \vec{u}) \models\left[\vec{X}=\vec{x}^{\prime}, \vec{W}=\vec{w}\right] \neg \varphi$.

(b) $(M, \vec{u}) \models\left[\vec{X}=\vec{x}, \vec{W}^{\prime}=\vec{w}, \vec{Z}^{\prime}=\vec{z}^{*}\right] \varphi$ for all subsets $\vec{W}^{\prime}$ of $\vec{W}$ and all subsets $\vec{Z}^{\prime}$ of $\vec{Z}$, where we abuse notation and write $\vec{W}^{\prime}=\vec{w}$ to denote the assignment where the variables in $\vec{W}^{\prime}$ get the same values as they would in the assignment $\vec{W}=\vec{w}$ (and similarly for $\vec{Z}$ ).

\footnotetext{
${ }^{6}$ In fact, this is actually labeled a preliminary definition in [Halpern and Pearl 2005], although it is very close the final definition. We ignore here the final modification, which will be supplanted by our new account. When we talk of "the HP definition", we refer to Definition 3.1 below, rather than to the final definition in [Halpern and Pearl 2005].
} 
AC3. $\vec{X}$ is minimal; no subset of $\vec{X}$ satisfies conditions AC1 and AC2.

AC1 just says that $\vec{X}=\vec{x}$ cannot be considered a cause of $\varphi$ unless both $\vec{X}=\vec{x}$ and $\varphi$ actually happen. AC3 is a minimality condition, which ensures that only those elements of the conjunction $\vec{X}=\vec{x}$ that are essential for changing $\varphi$ in AC2(a) are considered part of a cause; inessential elements are pruned. Without AC3, if dropping a lit match qualified as a cause of the forest fire, then dropping a match and sneezing would also pass the tests of AC1 and AC2. AC3 serves here to strip "sneezing" and other irrelevant, over-specific details from the cause. Clearly, all the "action" in the definition occurs in AC2. We can think of the variables in $\vec{Z}$ as making up the "causal path" from $\vec{X}$ to $\varphi$. Intuitively, changing the value of some variable(s) in $\vec{X}$ results in changing the value(s) of some variable(s) in $\vec{Z}$, which results in the values of some other variable(s) in $\vec{Z}$ being changed, which finally results in the truth value of $\varphi$ changing. The remaining endogenous variables, the ones in $\vec{W}$, are off to the side, so to speak, but may still have an indirect effect on what happens. AC2(a) is essentially the standard counterfactual definition of causality, but with a twist. If we want to show that $\vec{X}=\vec{x}$ is a cause of $\varphi$, we must show (in part) that if $\vec{X}$ had a different value, then so too would $\varphi$. However, this effect of the value of $\vec{X}$ on the value of $\varphi$ may not hold in the actual context; it may be necessary to intervene on the value of one or more variables in $\vec{W}$ to allow this effect to manifest itself. For example, consider the context where both the lightning strikes and the arsonist drops a match in the disjunctive model of the forest fire. Stopping the arsonist from dropping the match will not prevent the forest fire. The counterfactual effect of the arsonist on the forest fire manifests itself only in a situation where the lightning does not strike (i.e., where $L$ is set to 0 ). AC2(a) is what allows us to call both the lightning and the arsonist causes of the forest fire.

AC2(b) is perhaps the most complicated condition. It limits the "permissiveness" of AC2(a) with regard to the contingencies that can be considered. Essentially, it ensures that the change in the value $\vec{X}$ alone suffices to bring about the change from $\varphi$ to $\neg \varphi$; setting $\vec{W}$ to $\vec{w}$ merely eliminates possibly spurious side effects that may mask the effect of changing the value of $\vec{X}$. Moreover, although the values of variables on the causal path (i.e., the variables $\vec{Z}$ ) may be perturbed by the intervention on $\vec{W}$, this perturbation has no impact on the value of $\varphi$. We capture the fact that the perturbation has no impact on the value of $\varphi$ by saying that if some variables $Z$ on the causal path were set to their original values in the context $\vec{u}, \varphi$ would still be true, as long as $\vec{X}=\vec{x}$. Note that it follows from AC2(b) that an intervention that sets the value of the variables in $\vec{W}$ to their actual values is always permissible. Such an intervention might still constitute a change to the model, since the value of one or more variables in $\vec{W}$ might otherwise change when we change the value of $\vec{X}$ from $\vec{x}$ to $\vec{x}^{\prime}$. Note also that if $\varphi$ counterfactually depends on $\vec{X}=\vec{x}$, AC rules that $\vec{X}=\vec{x}$ is an actual cause of $\varphi$ (assuming that $\mathrm{AC} 1$ and $\mathrm{AC} 3$ are both satisfied). We can simply take $\vec{W}=\emptyset$; both clauses of AC2 are satisfied.

As we suggested in the introduction, a number of other definitions of actual causality follow a similar recipe (e.g., [Glymour and Wimberly 2007; Pearl 2000; Hitchcock 2001; Woodward 2003]). In order to show that $\dot{X}=\vec{x}$ is an actual cause of $\varphi$, first make a "permissible" modification of the causal model. Then, show that in the modified model, setting $\vec{X}$ to some different value $\vec{x}^{\prime}$ 
leads to $\varphi$ being false. The accounts differ in what counts as a permissible modification. For purposes of discussion, however, it is helpful to have one specific definition on the table; hence we will focus on the HP definition.

We now show how the HP definition handles our two problematic cases.

Example 3.2: For the forest-fire example, let $M$ be the disjunctive causal model for the forest fire sketched earlier, with endogenous variables $L, M$, and $F F$, and equation $F F=$ $\max (L, M)$. Clearly $(M,(1,1)) \models F F=1$ and $(M,(1,1)) \models L=1$; in the context $(1,1)$, the lightning strikes and the forest burns down. Thus, AC1 is satisfied. AC3 is trivially satisfied, since $\vec{X}$ consists of only one element, $L$, so must be minimal. For AC2, take $\vec{Z}=\{L, F F\}$ and take $\vec{W}=\{M\}$, let $x^{\prime}=0$, and let $w=0$. Clearly, $(M,(1,1)) \models[L=0, M=0](F F \neq 1)$; if the lightning does not strike and the match is not dropped, the forest does not burn down, so AC2(a) is satisfied. To see the effect of the lightning, we must consider the contingency where the match is not dropped; the definition allows us to do that by setting $M$ to 0 . (Note that here setting $L$ and $M$ to 0 overrides the effects of $U$; this is critical.) Moreover, $(M,(1,1)) \models[L=$ $1, M=0](F F=1)$; if the lightning strikes, then the forest burns down even if the lit match is not dropped, so AC2(b) is satisfied. (Note that since $\vec{Z}=\{L, F F\}$, the only subsets of $\vec{Z}-\vec{X}$ are the empty set and the singleton set consisting of just $F F$.) As this example shows, the HP definition need not pick out a unique actual cause; there may be more than one actual cause of a given outcome.7

Example 3.3: For the poisoning example, we can include in our causal model $M$ the following endogenous variables:

- $A=1$ if the assassin poisons the drink, 0 if not;

- $R=1$ if the backup is ready to poison the drink if necessary, 0 if not;

- $B=1$ if the backup poisons the drink, 0 if not;

- $D=1$ if the victim dies, 0 if not.

We also have an exogenous variable $U$ that determines whether the first assassin poisons the drink and whether the second assassin is ready. Let $U$ have four values of the form $\left(u_{1}, u_{2}\right)$ with $u_{i} \in\{0,1\}$ for $i=1,2$. The equations are

$$
\begin{aligned}
& A=u_{1} ; \\
& R=u_{2} ; \\
& B=(1-A) \times R ; \\
& D=\max (A, B) .
\end{aligned}
$$

\footnotetext{
${ }^{7}$ Of course, this is a familiar property of many theories of causation, such as Lewis's counterfactual definition [Lewis 1973].
} 
The third equation says that the backup poisons the drink if she is ready and the first assassin doesn't poison the drink. The fourth equation says that the victim dies if either assassin poisons the drink. This model is represented graphically in Figure 2. In the actual context, where $U=(1,1)$, we have $A=1, R=1, B=0$, and $D=1$. We want our account to give the result that $A=1$ is an actual cause of $D=1$, while $R=1$ is not.

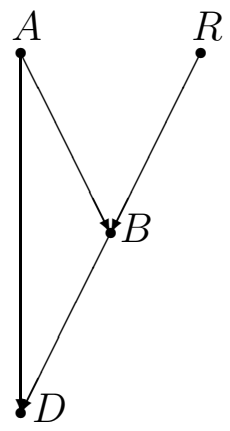

Figure 2: The poisoning example.

For the former, note that $D=1$ does not counterfactually depend on $A=1$ : if $A$ had been $0, B$ would have been 1 , and $D$ would still have been 1 . Nonetheless, Definition AC rules that $A=1$ is an actual cause of $D=1$. It is easy to see that $\mathrm{AC} 1$ and $\mathrm{AC} 3$ are satisfied. $A=1$ and $D=1$ are both true in the actual context where $U=(1,1)$, so AC1 is satisfied. Moreover, $A=1$ is minimal, so AC3 is satisfied. For $\mathrm{AC} 2$, let $\vec{Z}=\{A, D\}$ and $\vec{W}=\{R, B\}$, with $\vec{x}^{\prime}=0$ and $\vec{w}=(1,0)$ (i.e., $R=1$ and $\left.B=0\right)$. Checking AC2(a), we see that $(M,(1,1)) \models$ $[A=0, R=1, B=0](D \neq 1)$. That is, when we intervene to set $A$ to $0, R$ to 1 , and $B$ to $0, D$ takes a different value. While the victim's death does not counterfactually depend on the assassin's poisoning the drink, counterfactual dependence is restored if we also hold fixed that the backup did not act. Moreover, $\mathrm{AC} 2(\mathrm{~b})$ is also satisfied, for $\vec{W}=\vec{w}$ is just the setting $R=1$ and $B=0$, the values of $R$ and $B$ in the actual context. So if $A$ also takes on its actual value of 1 , then $D=1$. Note that we could also choose $R=0$ and $B=0$ for the setting $\vec{W}=\vec{w}$. That is, it makes no difference to either part of AC2 if we intervene to prevent the backup from being ready. Alternately, we could include $R$ in $\vec{Z}$ instead of $\vec{W}$; again the analysis is unaffected.

We now show that $R=1$ is not an actual cause of $D=1$. AC1 and AC3 are satisfied. In order to satisfy $\mathrm{AC} 2$ (a), we need $\vec{x}^{\prime}=0, \vec{Z}=\{R, B, D\}, \vec{W}=\{A\}$, and $\vec{w}^{\prime}=0$. In words, the only way to get the victim's death to counterfactually depend on the backup's readiness is if we intervene to prevent the first assassin from poisoning the drink. But now we can verify that these settings do not also satisfy AC2(b). Since the actual value of $B$ was $0, A C 2(b)$ requires that in order for $A=0$ to be an admissible setting of $\vec{W}=\{A\}$, we must have $(M,(1,1)) \models[A=0, B=0] D=1$. That is, in order for $A=0$ to be an admissible setting, this setting must not change the value of $D$, even if variables such as $B$ that are on the causal path from $R$ to $D$ are held fixed at their actual value. But this condition fails: $(M,(1,1)) \models[A=0, B=0] D=0$. Holding fixed that the backup did not poison the 
drink, if the assassin hadn't poisoned the drink either, the victim would have not have died. Intuitively, the idea is that the backup's readiness can be an actual cause of death only if the backup actually put poison in the drink. In this way, clause AC2(b) builds in the idea that there must be an appropriate sort of causal chain or process in order for $R=1$ to be an actual cause of $D=1$. This example also shows the importance of restricting the permissible contingencies that we can look at when re-evaluating counterfactual dependence.

\section{The Problem of Isomorphism}

In this section, we provide two illustrations of the problem of isomorphism, which was briefly mentioned in the introduction. Specifically, we will provide two examples in which the structure of counterfactual dependence is isomorphic to the structures in Examples 3.2 and 3.3. The first is an example of "bogus prevention", taken from Hitchcock [2007], based on an example due to Hiddleston [2005].

Example 4.1: Assassin is in possession of a lethal poison, but has a last-minute change of heart and refrains from putting it in Victim's coffee. Bodyguard puts antidote in the coffee, which would have neutralized the poison had there been any. Victim drinks the coffee and survives. Is Bodyguard's putting in the antidote a cause of Victim surviving? Most people would say no, but according to the HP definition, it is. For in the contingency where Assassin puts in the poison, Victim survives iff Bodyguard puts in the antidote.

The structural equations for Example 4.1 are isomorphic to those in the disjunctive version of the forest-fire example (example 3.2), provided that we interpret the variables appropriately. Specifically, take the endogenous variables in Example 4.1 to be $A$ (for "assassin does not put in poison"), $B$ (for "bodyguard puts in antidote"), and $V S$ (for "victim survives"). Then $A, B$, and $V S$ satisfy exactly the same equations as $L, M$, and $F F$, respectively. In the context where there is lightning and the arsonists drops a lit match, both the the lightning and the match are causes of the forest fire, which seems reasonable. But here it does not seem reasonable that Bodyguard's putting in the antidote is a cause. Nevertheless, any definition that just depends on the structural equations is bound to give the same answers in these two examples.

A second type of case illustrating the same problem involves what Hall [2007] calls "short circuits". Hitchcock [2007] gives the following example (which he calls " $\mathrm{CH}^{2}$ or "counterexample to Hitchcock", since it is a counterexample to the theory of [Hitchcock 2001]):

Example 4.2: A victim has two bodyguards charged with protecting him from assassination. The bodyguards conspire to make it appear as though they have foiled an attempted poisoning. They plan to put poison in victim's drink, and also to put in an antidote that will neutralize the poison. However, they do not want to put the poison in until they are certain that the antidote has safely been put in the drink. Thus, the first bodyguard adds antidote to the drink, and the second waits until the antidote has been added before adding the poison. If the first bodyguard 
were interrupted, or somehow prevented from putting the antidote in, the second would not add the poison. As it happens, both the antidote and the poison are added, so the poison is neutralized; the victim drinks the harmless liquid and lives.

Most people, although by no means all, judge that putting the antidote into the drink is not an actual cause of the victim's survival. Put another way, administering the antidote did not prevent death. This is because putting the antidote in the drink caused the very threat that it was meant to negate. If the antidote weren't put in, there would have been no poison to neutralize. However, it turns out that this example has a structure that is isomorphic to the preemption example discussed earlier (Example 3.3). This is not immediately obvious; we discuss the technical details in Section 7.7. For now, it suffices to note that if we hold fixed that the second bodyguard puts in the poison, then intervening on whether the first bodyguard puts in the antidote makes a difference for whether the victim dies.

In both kinds of case, examples that have isomorphic structural equation models are judged to have different relations of actual causation. This is not just a problem for the HP definition of actual causation. The problem of isomorphism affects any account of actual causation that appeals only to the structural equations in a causal model (or that appeals only to the structure of counterfactual dependence relations). This suggests that there must be more to actual causation than just the structural equations.

\section{Defaults, Typicality, and Normality}

Our revised account of actual causation incorporates the concepts of defaults, typicality, and normality. These are related, although somewhat different notions:

- A default is an assumption about what happens, or what is the case, when no additional information is given. For example, we might have as a default assumption that birds fly. If we are told that Tweety is a bird, and given no further information about Tweety, then it is natural to infer that Tweety flies. Such inferences are defeasible: they can be overridden by further information. If we are additionally told that Tweety is a penguin, we retract our conclusion that Tweety flies. Default logics (see, e.g., [Marek and Truszczyński 1993; Reiter 1980; Reiter 1987]) attempt to capture the structure of these kinds of inferences.

- To say that birds typically fly is to say not merely that flight is statistically prevalent among birds, but also that flight is characteristic of the type "bird". Even though not all birds do fly, flying is something that we do characteristically associate with birds.

- The word normal is interestingly ambiguous. It seems to have both a descriptive and a prescriptive dimension. To say that something is normal in the descriptive sense is to say that it is the statistical mode or mean (or close to it). On the other hand, we often use the shorter form norm in a more prescriptive sense. To conform with a norm is to follow 
a prescriptive rule. Prescriptive norms can take many forms. Some norms are moral: to violate them would be to perform a moral wrong. For example, many people believe that there are situations in which it would be wrong to lie, even if there are no laws or explicit rules forbidding this behavior. Laws are another kind of norm, adopted for the regulation of societies. Policies that are adopted by institutions can also be norms. For instance, a company may have a policy that employees are not allowed to be absent from work unless they have a note from their doctor. There can also be norms of proper functioning in machines or organisms. There are specific ways that human hearts and car engines are supposed to work, where "supposed" here has not merely an epistemic force, but a kind of normative force. Of course, a car engine that does not work properly is not guilty of a moral wrong, but there is nonetheless a sense in which it fails to live up to a certain kind of standard.

While this might seem like a heterogeneous mix of concepts, they are intertwined in a number of ways. For example, default inferences are successful just to the extent that the default is normal in the statistical sense. Adopting the default assumption that a bird can fly facilitates successful inferences in part because most birds are able to fly. Similarly, we classify objects into types in part to group objects into classes most of whose members share certain features. Thus, the type "bird" is useful partly because there is a suite of characteristics shared by most birds, including the ability to fly. The relationship between the statistical and prescriptive senses of "normal" is more subtle. It is, of course, quite possible for a majority of individuals to act in violation of a given moral or legal norm. Nonetheless, we think that the different kinds of norm often serve as heuristic substitutes for one another. For example, well-known experiments by Kahneman and Tversky [Kahneman and Tversky 1982; Tversky and Kahneman 1973] show that we are often poor at explicit statistical reasoning, employing instead a variety of heuristics. Rather than tracking statistics about how many individuals behave in a certain way, we might well reason about how people ought to behave in certain situations. The idea is that we use a script or a template for reasoning about certain situations, rather than actual statistics. Prescriptive norms of various sorts can play a role in the construction of such scripts. It is true, of course, that conflation of the different sorts of norm can sometimes have harmful consequences. Less than a hundred years ago, for example, left-handed students were often forced to learn to write with their right hands. In retrospect, this looks like an obviously fallacious inference from the premise that the majority of people write with their right hand to the conclusion that it is somehow wrong to write with the left hand. But the very ease with which such an inference was made illustrates the extent to which we find it natural to glide between the different senses of "norm".

That there should be a connection between defaults, norms, typicality, and causality is not a new observation. Kahneman and Tversky [1982], Kahneman and Miller [1986], and others have shown that both statistical and prescriptive norms can affect counterfactual reasoning. For example, Kahneman and Miller [1986, p. 143] point out that "an event is more likely to be undone by altering exceptional than routine aspects of the causal chain that led to it." Given the close connection between counterfactual reasoning and causal reasoning, this suggests that 
norms will also affect causal judgment. Recent experiments have confirmed this. Alicke [1992] and Alicke et al. [2011] show that subjects are more likely to judge that someone caused some negative outcome when they have a negative evaluation of that person. Cushman, Knobe, and Sinnott-Armstrong [2008] have shown that subjects are more likely to judge that an agent's action causes some outcome when they hold that the action is morally wrong; Knobe and Fraser [2008] have shown that subjects are more likely to judge that an action causes some outcome if it violates a policy; and Hitchcock and Knobe [2009] have shown that this effect occurs with norms of proper functioning.

Many readers are likely to be concerned that incorporating considerations of normality and typicality into an account of actual causation will have a number of unpalatable consequences. Causation is supposed to be an objective feature of the world. But while statistical norms are, arguably, objective, other kinds of norm do not seem to be. More specifically, the worry is that the incorporation of norms will render causation: (1) subjective, (2) socially constructed, (3) value-laden, (4) context-dependent, and (5) vague. It would make causation subjective because different people might disagree about what is typical or normal. For example, if moral values are not objective, then any effect of moral norms on causation will render causation subjective. Since some norms, such as laws, or policies implemented within institutions, are socially constructed, causation would become socially constructed too. Since moral norms, in particular, incorporate values, causation would become value-laden. Since there are many different kinds of norm, and they may sometimes come into conflict, conversational context will sometimes have to determine what is considered normal. This would render causation context-dependent. Finally, since typicality and normality seem to admit of degrees, this would render causation vague. But causation should be none of these things.

We believe that these worries are misplaced. While our account of actual causation incorporates all of these elements, actual causation is the wrong place to look for objectivity. Causal structure, as represented in the equations of a causal model, is objective. More precisely, once a suitable set of variables has been chosen, 8 there is an objectively correct set of structural equations among those variables. Actual causation, by contrast, is a fairly specialized causal notion. It involves the post hoc attribution of causal responsibility for some outcome, and is particularly relevant to making determinations of moral or legal responsibility. The philosophy literature tends to identify causation in general with actual causation. We believe that this identification is inappropriate. The confusion arises, in part, from the fact that in natural language we express judgments of actual causation using the simple verb "cause". We say, for example, that the lightning caused the fire, the assassin's poisoning the drink caused the victim to die, and that one neuron's firing caused another to fire. Our language gives us no clue that it is a rather specialized causal notion that we are deploying.

As we mentioned earlier, normality can be used to represent many different kinds of considerations. Further empirical research should reveal in greater detail just what kinds of factors can influence judgments of actual causation. For example, Knobe and Fraser [2008] and Roxborough and Cumby [2009] debate the relative importance of prescriptive norms and statistical

\footnotetext{
${ }^{8}$ See [Halpern and Hitchcock 2010] for discussion of what makes for a suitable choice of variables
} 
frequency, and what happens when these considerations pull in opposite directions. Sytsma et al. [2010] ask whether it is behavior that is typical for an individual, or behavior that is typical for a population to which that individual belongs, that affects judgments of actual causation, and how these factors interact.

Analogously, further philosophical debate may concern which factors ought to influence judgments of actual causation. For example, Hitchcock and Knobe [2009] argue that attributions of actual causation typically serve to identify appropriate targets of corrective intervention. Given the role it is supposed to play, it is not at all inappropriate for normative considerations to play a role in judgments of actual causation. A closely related suggestion is developed by Lombrozo [2010]. She argues that judgments of actual causation identify dependence relations that are highly portable into other contexts. For this reason, it makes sense to focus on factors that make a difference for the intended outcome in normal circumstances. (See also Danks [2013] and Hitchcock [2012] for further discussion.) We do not intend for our formal framework to prejudge these kinds of issues. Rather, we intend it to be flexible enough to represent whatever considerations of normality or default behavior are deemed appropriate to judgments of actual causation in a particular context.

Even for those hard-nosed metaphysicians who eschew any appeal to subjective considerations of normality, we think that our formal framework can have something to offer. For example, Moore [2009] argues that causation is a relation between events, where an event is a change in the state of some system, and not just a standing state. Thus, a lightning strike may be an event, but the presence of oxygen in the atmosphere is not. Similarly, Glymour et al. [2010] suggest that judgments of actual causation will depend upon the starting conditions of a system. This kind of idea can be represented by taking the state of a system at some "start time" to be the default state of the system. Changes in the system will then result in states that are atypical. A closely related idea is developed (in different ways) by Hitchcock [2007], Maudlin [2004], and Menzies [2004, 2007]. Here, instead of taking the absence of change to be the default, one takes a certain evolution of an "undisturbed" system to be its default behavior. The classic example is from Newtonian mechanics: the default behavior of a body is to continue in a state of uniform motion.

While there are a large number of factors that can influence what constitutes normal or default behavior, and at least some of these considerations will be subjective, context-dependent, and so on, we do not think that the assignment of norms and defaults is completely unconstrained. When a causal model is being used in some context, or for some specific purpose, certain assignments of default values to variables are more natural, or better motivated, than others. An assignment of norms and defaults is the sort of thing that can be defended and criticized on rational grounds. For example, if a lawyer were to argue that a defendant did or did not cause some harm, she would have to give arguments in support of her assignments of norms or defaults. 


\section{Extended Causal Models}

Following Halpern [2008], we deal with the problems raised in Section 4 by assuming that an agent has, in addition to a theory of causal structure (as modeled by the structural equations), a theory of "normality" or "typicality". This theory would include statements like "typically, people do not put poison in coffee". There are many ways of giving semantics to such typicality statements, including preferential structures [Kraus, Lehmann, and Magidor 1990; Shoham 1987], $\epsilon$-semantics [Adams 1975; Geffner 1992; Pearl 1989], possibilistic structures [Dubois and Prade 1991], and ranking functions [Goldszmidt and Pearl 1992; Spohn 1988]. Halpern [2008] used the last approach, but it builds in an assumption that the normality order on worlds is total. We prefer to allow for some worlds to be incomparable. This seems plausible, for example, if different types of typicality or normality are in play; or if we are comparing the normality of different kinds of behavior by different people or objects. (This also plays a role in our treatment of bogus prevention in Section 7.4.) Thus, we use a slightly more general approach here, based on preferential structures.

Take a world to be an assignment of values to all the exogenous variables in a causal model 9 Intuitively, a world is a complete description of a situation given the language determined by the set of endogenous variables. Thus, a world in the forest-fire example might be one where $M=1, L=0$, and $F F=0$; the match is dropped, there is no lightning, and no forest fire. As this example shows, a "world" does not have to satisfy the equations of the causal model.

For ease of exposition, in the rest of the paper, we make a somewhat arbitrary stipulation regarding terminology. In what follows, we use "default" and "typical" when talking about individual variables or equations. For example, we might say that the default value of a variable is zero, or that one variable typically depends on another in a certain way. We use "normal" when talking about worlds. Thus, we say that one world is more normal than another. In the present paper, we do not develop a formal theory of typicality, but assume only that typical values for a variable are influenced by the kinds of factors discussed in the previous section. We also assume that it is typical for endogenous variables to be related to one another in the way described by the structural equations of a model, unless there is some specific reason to think otherwise. The point of this assumption is to ensure that the downstream consequences of what is typical are themselves typical (again, in the absence of any specific reason to think otherwise).

In contrast to our informal treatment of defaults and typicality, we provide a formal representation of normality. We assume that there is a partial preorder $\succeq$ over worlds; $s \succeq s^{\prime}$ means that world $s$ is at least as normal as world $s^{\prime}$. The fact that $\succeq$ is a partial preorder means that it is reflexive (for all worlds $s$, we have $s \succeq s$, so $s$ is at least as normal as itself) and transitive (if $s$ is at least as normal as $s^{\prime}$ and $s^{\prime}$ is at least as normal as $s^{\prime \prime}$, then $s$ is at least as normal as

\footnotetext{
9' might be apt to use "small world" to describe such an assignment, to distinguish it from a "large world", which would be an assignment of values to all of the variables in a model, both endogenous and exogenous. While there may well be applications where large worlds are needed, the current application requires only small worlds. The reason for this is that all of the worlds that are relevant to assessing actual causation in a specific context $\vec{u}$ result from intervening on endogenous variables, while leaving the exogenous variables unchanged.
} 
$\left.s^{\prime \prime}\right) .10$ We write $s \succ s^{\prime}$ if $s \succeq s^{\prime}$ and it is not the case that $s^{\prime} \succeq s$, and $s \equiv s^{\prime}$ if $s \succeq s^{\prime}$ and $s^{\prime} \succeq s$. Thus, $s \succ s^{\prime}$ means that $s$ is strictly more normal than $s^{\prime}$, while $s \equiv s^{\prime}$ means that $s$ and $s^{\prime}$ are equally normal. Note that we are not assuming that $\succeq$ is total; it is quite possible that there are two worlds $s$ and $s^{\prime}$ that are incomparable as far as normality. The fact that $s$ and $s^{\prime}$ are incomparable does not mean that $s$ and $s^{\prime}$ are equally normal. We can interpret it as saying that the agent is not prepared to declare either $s$ or $s^{\prime}$ as more normal than the other, and also not prepared to say that they are equally normal; they simply cannot be compared in terms of normality.

One important issue concerns the relationship between typicality and normality. Ideally, one would like to have a sort of compositional semantics. That is, given a set of statements about the typical values of particular variables and a causal model, a normality ranking on worlds could be generated that in some sense respects those statements. We develop such an account in a companion paper [Halpern and Hitchcock 2012]. In the present paper, we make do with a few rough-and-ready guidelines. Suppose that $s$ and $s^{\prime}$ are worlds, that there is some nonempty set $\vec{X}$ of variables that take more typical values in $s$ than they do in $s^{\prime}$, and no variables that take more typical values in $s^{\prime}$ than in $s$; then $s \succ s^{\prime}$. However, if there is both a nonempty $\vec{X}$ set of variables that take more typical values in $s$ than they do in $s^{\prime}$, and a nonempty set $\vec{Y}$ of variables that take more typical values in $s^{\prime}$ than they do in $s$, then $s$ and $s^{\prime}$ are incomparable, unless there are special considerations that would allow us to rank them. This might be in the form of statement that $\vec{x}$ is a more typical setting for $\vec{X}$ than $\vec{y}$ is of $\vec{Y}$. We consider an example where such a rule seems very natural in Section 7.6 below.

Take an extended causal model to be a tuple $M=(\mathcal{S}, \mathcal{F}, \succeq)$, where $(\mathcal{S}, \mathcal{F})$ is a causal model, and $\succeq$ is a partial preorder on worlds, which can be used to compare how normal different worlds are. In particular, $\succeq$ can be used to compare the actual world to a world where some interventions have been made. Which world is the actual world? That depends on the context. In an acyclic extended causal model, a context $\vec{u}$ determines a world denoted $s_{\vec{u}}$. We can think of the world $s_{\vec{u}}$ as the actual world, given context $\vec{u}$, since it is the world that would occur given the setting of the exogenous variables in $\vec{u}$, provided that there are no external interventions.

Unlike the treatments developed by Huber [2011] and Menzies [2004, 2007], the ordering $\succeq$ does not affect which counterfactuals are true. This is determined by the equations of the causal model. $\succeq$ does not determine which worlds are 'closer' than others for purposes of evaluating counterfactuals. We envision a kind of conceptual division of labor, where the causal model $(\mathcal{S}, \mathcal{F})$ represents the objective patterns of dependence that could in principle be tested by intervening on a system, and $\succeq$ represents the various normative and contextual factors that also influence judgments of actual causation.

We can now modify Definition 3.1 slightly to take the ranking of worlds into account by taking $\vec{X}=\vec{x}$ to be a cause of $\varphi$ in an extended model $M$ and context $\vec{u}$ if $\vec{X}=\vec{x}$ is a cause of $\varphi$ according to Definition 3.1, except that in AC2(a), we add a clause requiring that

\footnotetext{
${ }^{10}$ If $\succeq$ were a partial order rather than just a partial preorder, it would satisfy an additional assumption, antisymmetry: $s \succeq s^{\prime}$ and $s^{\prime} \succeq s$ would have to imply $s=s^{\prime}$. This is an assumption we do not want to make.
} 
$s_{\vec{X}=\vec{x}^{\prime}, \vec{W}=\vec{w}, \vec{u}} \succeq s_{\vec{u}}$, where $s_{\vec{X}=\vec{x}^{\prime}, \vec{W}=\vec{w}, \vec{u}}$ is the world that results by setting $\vec{X}$ to $\vec{x}^{\prime}$ and $\vec{W}$ to $\vec{w}$ in context $\vec{u}$. This can be viewed as a formalization of Kahneman and Miller's [1986] observation that we tend to consider only possibilities that result from altering atypical features of a world to make them more typical, rather than vice versa. In our formulation, worlds that result from interventions on the actual world "come into play" in AC2(a) only if they are at least as normal as the actual world.

In addition, we can use normality to rank actual causes. Doing so lets us explain the responses that people make to queries regarding actual causation. For example, while counterfactual approaches to causation usually yield multiple causes of an outcome $\varphi$, people typically mention only one of them when asked for a cause. We would argue that they are picking the best cause, where best is judged in terms of normality. We now make this precise.

Say that world $s$ is a witness for $\vec{X}=\vec{x}$ being a cause of $\varphi$ in context $\vec{u}$ if for some choice of $\vec{Z}, \vec{W}, \vec{x}^{\prime}$, and $\vec{w}$ for which AC2(a) and AC2(b) hold, $s$ is the assignment of values to the endogenous variables that results from setting $\vec{X}=\vec{x}^{\prime}$ and $\vec{W}=\vec{w}$ in context $\vec{u}$. In other words, a witness $s$ is a world that demonstrates that AC2(a) holds. In general, there may be many witnesses for $\vec{X}=\vec{x}$ being a cause of $\varphi$. Say that $s$ is a best witness for $\vec{X}=\vec{x}$ being a cause of $\varphi$ if there is no other witness $s^{\prime}$ such that $s^{\prime} \succ s$. (Note that there may be more than one best witness.) We can then grade candidate causes according to the normality of their best witnesses. We expect that someone's willingness to judge that $\vec{X}=\vec{x}$ is an actual cause of $\varphi$ increases as a function of the normality of the best witness for $\vec{X}=\vec{x}$ in comparison to the best witness for other candidate causes. Thus, we are less inclined to judge that $\vec{X}=\vec{x}$ is an actual cause of $\varphi$ when there are other candidate causes of equal or higher rank.

This strategy of ranking causes according to the normality of their best witness can be adapted to any definition of actual cause that has the same basic structure as the HP definition. Consider, for example, the proposal of Hall [2007]. In order to show that $\vec{X}=\vec{x}$ is an actual cause of $\varphi$ in model $M$ with context $\vec{u}$, Hall requires that we find a suitable context $\vec{u}^{\prime}$, such that $\left(M, \vec{u}^{\prime}\right)$ is a reduction of $(M, \vec{u})$. The precise definition of a reduction is not important for the present illustration. Then one must show that changing the value of $\vec{X}$ from $\vec{x}$ to $\vec{x}^{\prime}$ changes the truth value of $\varphi$ from true to false in $\left(M, \vec{u}^{\prime}\right)$. We can then call the possible world that results setting $\vec{X}$ to $\vec{x}^{\prime}$ in $\left(M, \vec{u}^{\prime}\right)$ a witness; it plays exactly the same role in Hall's definition that a witness world plays in the HP definition. We can then use a normality ordering on worlds to rank causes according to their most normal witness.

\section{Examples}

In this section, we give a number of examples of the power of this definition. (For simplicity, we omit explicit reference to the exogenous variables in the discussions that follow.) 


\subsection{Omissions}

As we mentioned in the introduction, there is disagreement in the literature over whether causation by omission should count as actual causation, despite the fact that there is no disagreement regarding the underlying causal structure. We can distinguish (at least) four different viewpoints in the flower-watering example described in the introduction:

(a) Beebee [2004] and Moore [2009], for example, argue against the existence of causation by omission in general;

(b) Lewis [2000, 2004] and Schaffer [2000, 2004] argue that omissions are genuine causes in such cases;

(c) Dowe [2000] and Hall [2004] argue that omissions have a kind of secondary causal status; and

(d) McGrath [2005] argues that the causal status of omissions depends on their normative status: whether the neighbor's omission caused the flowers to die depends on whether the neighbor was supposed to water the flowers.

Our account of actual causation can accommodate all four viewpoints, by making suitable changes in the normality ordering. The obvious causal structure has three endogenous variables:

- $H=1$ if the weather is hot, 0 if it is cool;

- $W=1$ if the neighbor waters the flowers, 0 otherwise;

- $D=1$ if the flowers die, 0 if they do not.

There is one equation:

$$
D=H \times(1-W) .
$$

The exogenous variables are such that $H=1$ and $W=0$, hence in the actual world, $H=1$, $W=0$, and $D=1$. The original HP definition rules that both $H=1$ and $W=0$ are actual causes of $D=1$. The witness for $H=1$ being a cause is the world $(H=0, W=0, D=0)$, while the witness for $W=0$ is $(H=1, W=1, D=0)$. We claim that the difference between the viewpoints mentioned above can be understood as a disagreement either about the appropriate normality ranking, or the effect of graded causality.

Those who maintain that omissions are never causes can be understood as having a normality ranking where absences or omissions are more typical than positive events. That is, the typical value for both $H$ and $W$ is 0 . (However, $D$ will typically be 1 when $H=1$ and $W=0$, in accordance with the equation.) This ranking reflects a certain metaphysical view: there is a fundamental distinction between positive events and mere absences, and in the context of 
causal attribution, absences are always considered typical for candidate causes. This gives rise to a normality ranking where

$$
(H=0, W=0, D=0) \succ(H=1, W=0, D=1) \succ(H=1, W=1, D=0) .
$$

The fact that $(H=0, W=0, D=0) \succ(H=1, W=0, D=1)$ means that we can take $(H=0, W=0, D=0)$ as a witness for $H=1$ being a cause of $D=1$. Indeed, most people would agree that the hot weather was a cause of the plants dying. Note that $(H=1, W=1, D=0)$ is the witness for $W=0$ being a cause of $D=1$. If we take the actual world $(H=1, W=0, D=1)$ to be more normal than this witness, intuitively, treating not acting as more normal than acting, then we cannot view $W=0$ as an actual cause.

It should be clear that always treating "not acting" as more normal than acting leads to not allowing causation by omission. However, one potential problem for this sort of view is that it is not always clear what counts as a positive event, and what as a mere omission. For example, is holding one's breath a positive event, or is it merely the absence of breathing? If an action hero survives an encounter with poison gas by holding her breath, is this a case of (causation by) omission?

An advocate of the third viewpoint, that omissions have a kind of secondary causal status, may be interpreted as allowing a normality ordering of the form

$$
(H=0, W=0, D=0) \succ(H=1, W=1, D=0) \succeq(H=1, W=0, D=1) .
$$

This theory allows watering the plants to be as normal as not watering them, and hence $W=0$ can be an actual cause of $D=1$. However, $H=1$ has a more normal witness, so under the comparative view, $H=1$ is a much better cause than $W=0$. On this view, then, we would be more strongly inclined to judge that $H=1$ is an actual cause than that $W=0$ is an actual cause. However, unlike those who deny that $W=0$ is a cause of any kind, advocates of the third position might maintain that since $W=0$ has a witness, it has some kind of causal status, albeit of a secondary kind.

An advocate of the second viewpoint, that omissions are always causes, could have essentially the same ordering as an advocate of the second viewpoint, but would take the gap between $(H=0, W=0, D=0)$ and $(H=1, W=1, D=0)$ to be much smaller, perhaps even allowing that $(H=0, W=0, D=0) \equiv(H=1, W=1, D=0)$. Indeed, if $(H=0, W=0, D=0) \equiv(H=1, W=1, D=0)$, then $H=1$ and $W=0$ are equally good candidates for being actual causes of $D=1$. But note that with this viewpoint, not only is the neighbor who was asked to water the plants but did not a cause, so are all the other neighbors who were not asked.

The fourth viewpoint is that the causal status of omissions depends on their normative status. For example, suppose the neighbor had promised to water the homeowners' flowers; or suppose the two neighbors had a standing agreement to water each others' flowers while the other was away; or that the neighbor saw the wilting flowers, knew how much the homeowner loved her flowers, and could have watered them at very little cost or trouble to herself. In any of these cases, we might judge that the neighbor's failure to water the flowers was a cause of their 
death. On the other hand, if there was no reason to think that the neighbor had an obligation to water the flowers, or no reasonable expectation that she would have done so (perhaps because she, too, was out of town), then we would not count her omission as a cause of the flowers' death.

On this view, the existence of a norm, obligation, or expectation regarding the neighbor's behavior has an effect on whether the world $(H=1, W=1, D=0)$ is considered at least as normal as the actual world $(H=1, W=0, D=1) 11$ This viewpoint allows us to explain why not all neighbors' failures to water the flowers should be treated equally.

\subsection{Knobe effects}

In a series of papers (e.g., [Cushman, Knobe, and Sinnott-Armstrong 2008; Hitchcock and Knobe 2009; Knobe and Fraser 2008]), Joshua Knobe and his collaborators have demonstrated that norms can influence our attributions of actual causation. For example, consider the following vignette, drawn from Knobe and Fraser [2008]:

The receptionist in the philosophy department keeps her desk stocked with pens. The administrative assistants are allowed to take pens, but faculty members are supposed to buy their own. The administrative assistants typically do take the pens. Unfortunately, so do the faculty members. The receptionist repeatedly e-mailed them reminders that only administrators are allowed to take the pens. On Monday morning, one of the administrative assistants encounters Professor Smith walking past the receptionist's desk. Both take pens. Later, that day, the receptionist needs to take an important message ... but she has a problem. There are no pens left on her desk.

Subjects were then randomly presented with one of the following propositions, and asked to rank their agreement on a seven point scale from -3 (completely disagree) to +3 (completely agree):

Professor Smith caused the problem.

The administrative assistant caused the problem.

Subjects gave an average rating of 2.2 to the first claim, indicating agreement, but -1.2 to the second claim, indicating disagreement. Thus subjects are judging the two claims differently, due to the different normative status of the two actions. (Note that subjects were only presented with one of these claims: they were not given a forced choice between the two.)

\footnotetext{
${ }^{11}$ Jonathan Livengood [personal communication] asked what would happen if the neighbor had been asked to water the flowers, but the neighbor is known to be unreliable, and hence would not be expected (in the epistemic sense) to water the flowers. Would the neighbor's unreliability mitigate our causal judgment? And if so, does this mean that one can avoid moral responsibility by cultivating a reputation for negligence? This is the kind of case where empirical investigation and normative analysis is needed to determine an appropriate normality ordering, as discussed at the end of Section 5. Our hunch is that while the neighbor may be no less morally culpable in such a case, people's attribution of actual causation may be diminished, since they will assign some causal responsibility to the homeowner for choosing such an unreliable person to tend the flowers in her absence.
} 
If subjects are presented with a similar vignette, but where both groups are allowed to take pens, then subjects tend to give intermediate values. That is, when the vignette is changed so that Professor Smith is not violating a norm when he takes the pen, not only are subjects less inclined to judge that Professor Smith caused the problem, but they are more inclined to judge that the administrative assistant caused the problem.12 This is interesting, since the status of the administrative assistant's action has not been changed. The most plausible interpretation of this result is that subjects' increased willingness to say that the administrative assistant caused the problem is a direct result of their decreased willingness to say that Professor Smith caused the problem. This suggests that attributions of actual causation are at least partly a comparative affair.

The obvious causal model of the original vignette has three random variables:

- $P T=1$ if Professor Smith takes the pen, 0 if she does not;

- $A T=1$ if the administrative assistant takes the pen, 0 if she does not;

- $P O=1$ if the receptionist is unable to take a message, 0 if no problem occurs.

There is one equation:

$$
P O=\min (P T, A T) .
$$

The exogenous variables are such that $P T$ and $A T$ are both 1 . Therefore, in the actual world, we have $P T=1, A T=1$, and $P O=1$.

The HP definition straightforwardly rules that $P T=1$ and $A T=1$ are both actual causes of $P O=1$. (In both cases, we can let $\vec{W}$ be the empty set.) The best witness for $P T=1$ being a cause is $(P T=0, A T=1, P O=0)$; the best witness for $A T=1$ being a cause is $(P T=1, A T=0, P O=0)$. The original story suggests that the witness for $P T=1$ being a cause is more normal than the witness for $A T=1$, since administrative assistants are allowed to take pens, but professors are supposed to buy their own. So our account predicts that we are more strongly inclined to judge that $P T=1$ is an actual cause. On the other hand, if the vignette does not specify that one of the actions violates a norm, we would expect the relative normality of the two witnesses to be much closer, which is reflected in how subjects actually rated the causes.

This analysis assumes that we can identify the relevant norms prior to assessing actual causation. For example, in Knobe's original example, there is a departmental policy stating who is allowed to take the pens. This allows us to judge that Professor Smith violated the norm without needing to know whether his action was a cause of the problem. However, if the norm were of the form "don't cause problems", we would not be in a position to determine if the norm had been violated until we had already made our judgment about actual causation.

\footnotetext{
${ }^{12}$ Sytsma, Livengood, and Rose [2010] conducted the experiments. They had their subjects rate their agreement on 7-point scale from 1 (completely disagree) to 7 (completely agree). When they repeated Knobe and Fraser's original experiment, they got a rating of 4.05 for Professor Smith, and 2.51 for the administrative assistant. While their difference is less dramatic than Knobe and Fraser's, it is still statistically significant. When they altered the vignette so that Professor Smith's action was permissible, subjects gave an average rating of 3.0 for Professor Smith, and 3.53 for the administrative assistant.
} 


\subsection{Causes vs. background conditions}

It is common to distinguish between causes of some outcome, and mere background conditions that are necessary for that outcome (e.g. [Hart and Honoré 1985]). A standard example is a fire that is caused by a lit match. While the fire would not have occurred without the presence of oxygen in the atmosphere, the oxygen is deemed to be a background condition, rather than a cause.

We have three variables:

- $M=1$ if the match is lit, 0 if it is not lit;

- $O=1$ if oxygen is present, 0 if there is no oxygen;

- $F=1$ if there is a fire, 0 if there is no fire.

There is one equation:

$$
F=\min (M, O) \text {. }
$$

The exogenous variables are such that in the actual world, $M=1$ and $O=1$, so $F=1$.

Again, $F=1$ counterfactually depends on both $M=1$ and $O=1$, so the HP definition rules that both are actual causes of $F=1$. The witness for $M=1$ being a cause is the world $(M=0, O=1, F=0)$; the witness for $O=1$ being a cause is $(M=1, O=0, F=0)$. The fact that we take the presence of oxygen for granted means that the normality ordering makes a world where oxygen is absent quite abnormal. In particular, we require that it satisfy the following properties:

$$
(M=0, O=1, F=0) \succeq(M=1, O=1, F=1) \succ(M=1, O=0, F=0) .
$$

The first world is the witness for $M=1$ being a cause of $F=1$, the second is the actual world, and the third is the witness for $O=1$ being a cause. Thus, we are inclined to judge $M=1 \mathrm{a}$ cause and not judge $O=1$ a cause.

Note that if the fire occurred in a special chamber in a scientific laboratory that is normally voided of oxygen, then we would have a different normality ordering. Now the presence of oxygen is atypical, and the witness for $O=1$ being a cause is as normal as (or at least not strictly less normal than) the witness for $M=1$ being a cause. And this corresponds with our intuition that, in such a case, we would be willing to judge the presence of oxygen an actual cause of the fire 13

\footnotetext{
${ }^{13}$ Jonathan Livengood [personal communication] has suggested that we might still consider the match to be the primary cause of the fire in such a case, because the match undergoes a change in state, while the presence of the oxygen is a standing condition. This fits with the suggestion, made at the end of Section 5 , that we might take the state of some system at a 'start' time to be its default state. In this case, the normality ordering would be the same as in the original example. As discussed in Section 5, our concern here is not with determining what the "right" normality ordering is, but with providing a flexible framework that can be used to represent a variety of factors that might influence judgments of actual causation.
} 
Our treatment of Knobe effects and background conditions is likely to produce a familiar complaint. It is common in discussions of causation to note that while people commonly do make these kinds of discriminations, it is in reality a philosophical mistake to do so. For example, John Stuart Mill writes:

It is seldom, if ever, between a consequent and a single antecedent, that this invariable sequence subsists. It is usually between a consequent and the sum of several antecedents; the concurrence of all of them being requisite to produce, that is, to be certain of being followed by, the consequent. In such cases it is very common to single out one only of the antecedents under the denomination of Cause, calling the others mere Conditions ... The real cause, is the whole of these antecedents; and we have, philosophically speaking, no right to give the name of cause to one of them, exclusively of the others. [Mill 1856, pp. 360-361].

David Lewis says:

We sometimes single out one among all the causes of some event and call it "the" cause, as if there were no others. Or we single out a few as the "causes", calling the rest mere "causal factors" or "causal conditions"... I have nothing to say about these principles of invidious discrimination. [Lewis 1973, pp. 558-559]

And Ned Hall adds:

When delineating the causes of some given event, we typically make what are, from the present perspective, invidious distinctions, ignoring perfectly good causes because they are not sufficiently salient. We say that the lightning bolt caused the forest fire, failing to mention the contribution of the oxygen in the air, or the presence of a sufficient quantity of flammable material. But in the egalitarian sense of "cause," a complete inventory of the fire's causes must include the presence of oxygen and of dry wood. [Hall 2004, p. 228]

The concern is that because a cause is not salient, or because it would be inappropriate to assert that it is a cause in some conversational context, we are mistakenly inferring that it is not a cause at all. In a similar vein, an anonymous referee worried that our account rules out the possibility that a typical event whose absence would be atypical could ever count as a cause.

The "egalitarian" notion of cause is entirely appropriate at the level of causal structure, as represented by the equations of a causal model. These equations represent objective features of the world, and are not sensitive to factors such as contextual salience. We think that it is a mistake, however, to look for the same objectivity in actual causation. Hitchcock and Knobe (2009) argue that it is in part because of its selectivity that the concept of actual causation earns its keep. The "standard" view, as illustrated by Lewis [1973], for example, is something like this: There is an objective structure of causal dependence relations among events, understood, for example, in terms of non-backtracking counterfactuals. From these relations, we can define 
a relation of actual causation, which is also objective. Pragmatic, subjective factors then determine which actual causes we select and label "the" causes. On our view, we also begin with an objective structure, which for us is represented by the causal model. However, for us, the pragmatic factors that influence causal selection also play a role in defining actual causation. In particular, the same discriminatory mechanisms that influence causal selection also serve to discriminate among isomorphic causal models. Both views agree that there is an underlying objective causal structure, and that pragmatic factors influence causal selection. We disagree only about which side of the line the concept of actual causation falls on.

\subsection{Bogus prevention}

Consider the bogus prevention problem of Example 4.1. Suppose that we use a causal model with three random variables:

- $A=1$ if Assassin does puts in the poison, 0 if he does not;

- $B=1$ if Bodyguard adds the antidote, 0 if he does not;

- $V S=1$ if the victim survives, 0 if he does not.

Then the equation for $V S$ is

$$
V S=\max ((1-A), B) .
$$

$A, B$, and $V S$ satisfy exactly the same equations as $1-L, M$, and $F F$, respectively in Example 3.2. In the context where there is lightning and the arsonists drops a lit match, both the the lightning and the match are causes of the forest fire, which seems reasonable. Not surprisingly, the original HP definition declares both $A=0$ and $B=1$ to be actual causes of $V S=1$. But here it does not seem reasonable that Bodyguard's putting in the antidote is a cause.

Using normality gives us a straightforward way of dealing with the problem. In the actual world, $A=0, B=1$, and $V S=1$. The witness for $B=1$ to be an actual cause of $V S=1$ is the world where $A=1, B=0$, and $V S=0$. If we make the assumption that both $A$ and $B$ typically take the value 0.14 and make the assumptions about the relation between typicality and normality discussed in Section 6, this leads to a normality ordering in which the two worlds $(A=0, B=1, V S=1)$ and $(A=1, B=0, V S=0)$ are incomparable. Since the unique witness for $B=1$ to be an actual cause of $V S=1$ is incomparable with the actual world, our modified definition rules that $B=1$ is not an actual cause of $V S=1$. Interestingly, our account also rules that $A=0$ is not an actual cause, since it has the same witness. This does not strike us as especially counterintuitive.

This example illustrates an advantage of the present account over the one offered in [Halpern 2008], in which normality is characterized by a total order. With a total order, we cannot declare

\footnotetext{
${ }^{14}$ Some readers have suggested that it would not be atypical for an assassin to poison the victim's drink. That is what assassins do, after all. Nonetheless, the action is morally wrong and unusual from the victim's perspective, both of which would tend to make it atypical.
} 
$(A=1, B=0, V S=0)$ and $(A=0, B=1, V S=1)$ to be incomparable; we must compare them. To argue $A=1$ is not a cause, we have to assume that $(A=0, B=1, V S=1)$ is more normal than $(A=1, B=0, V S=0)$. This ordering does not seem so natural 15

\subsection{Causal chains}

There has been considerable debate in the philosophical literature over whether causation is transitive, that is, whether whenever $A$ causes $B$, and $B$ causes $C$, then $A$ causes $C$. Lewis [2000], for example, defends the affirmative, while Hitchcock [2001] argues for the negative. But even those philosophers who have denied that causation is transitive in general have not questioned the transitivity of causation in simple causal chains, where the final effect counterfactually depends on the initial cause. By contrast, the law does not assign causal responsibility for sufficiently remote consequences of an action. For example, in Regina v. Faulkner [1877], a well-known Irish case, a lit match aboard a ship caused a cask of rum to ignite, causing the ship to burn, which resulted in a large financial loss by Lloyd's insurance, leading to the suicide of a financially ruined insurance executive. The executive's widow sued for compensation, and it was ruled that the negligent lighting of the match was not a cause (in the legally relevant sense) of his death. Moore [2009] uses this type of case to argue that our ordinary notion of actual causation is graded, rather than all-or-nothing, and that it can attenuate over the course of a causal chain.

Our account of actual causation can make sense of this kind of attenuation. We can represent the case of Regina v. Faulkner using a causal model with nine random variables:

- $M=1$ if the match is lit, 0 if it is not;

- $R=1$ if there is rum in the vicinity of the match, 0 if not;

- $R I=1$ if the rum ignites, 0 if it does not;

- $F=1$ if there is further flammable material near the rum, 0 if not;

- $S D=1$ if the ship is destroyed, 0 if not;

- $L I=1$ if the ship is insured by Lloyd's, 0 if not;

15 There is another, arguably preferable, way to handle this case using the original HP definition, without appealing to normality. Suppose we add a variable $P N$ to the model, representing whether a chemical reaction takes place in which poison is neutralized. The model has the following equations:

$$
\begin{aligned}
& P N=A \times B ; \\
& V S=\max ((1-A), P N) .
\end{aligned}
$$

It is isomorphic to the model in Example 3.3, except that $A$ and $1-A$ are reversed. In this new model, $B=1$ fails to be an actual cause of $V S=1$ for the same reason the backup's readiness was not a cause of the victim's death in Example 3.3 By adding $P N$ to the model, we can capture the intuition that the antidote doesn't count as a cause of survival unless it actually neutralized poison. 
- $L L=1$ if Lloyd's suffers a loss, 0 if not;

- $E U=1$ if the insurance executive was mentally unstable, 0 if not;

- $E S=1$ if the executive commits suicide, 0 if not.

There are four structural equations:

$$
\begin{aligned}
& R I=\min (M, R) \\
& S D=\min (R I, F) \\
& L L=\min (S D, L I) \\
& E S=\min (L L, E U) .
\end{aligned}
$$

This model is shown graphically in Figure 3. The exogenous variables are such that $M, R$, $F, L I$, and $E U$ are all 1 , so in the actual world, all variables take the value 1 . Intuitively, the events $M=1, R I=1, S D=1, L L=1$, and $E S=1$ form a causal chain. The HP definition rules that the first four events are all actual causes of $E S=1$.

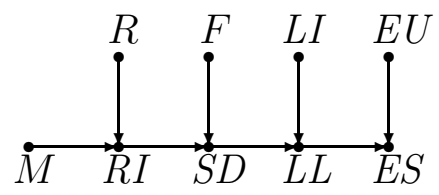

Figure 3: Attenuation in a causal chain.

Let us now assume that, for the variables $M, R, F, L I$, and $E U, 0$ is the typical value, and 1 is the atypical value. Thus, our normality ranking assigns a higher rank to worlds where more of these variables take the value 0 . For simplicity, consider just the most proximate and the most distal links in the chain: $L L=1$ and $M=1$, respectively. The world $(M=0, R=$ $1, R I=0, F=1, S D=0, L I=1, L L=0, E U=1, E S=0)$ is a witness of $M=1$ being a cause of $E S=1$. This is quite an abnormal world, although more normal than the actual world, so $M=1$ does count as an actual cause of $E S=1$ by our revised definition. Note that if any of the variables $R, F, L I$, or $E U$ is set to 0, then we no longer have a witness. Intuitively, $E S$ counterfactually depends on $M$ only when all of these other variables take the value 1 . Now consider the event $L L=1$. The world $(M=0, R=0, R I=0, F=0, S D=$ $0, L I=0, L L=0, E U=1, E S=0)$ is a witness for $L L=1$ being an actual cause of $E S=1$. This witness is significantly more normal than the best witness for $M=1$ being a cause. Intuitively, $L L=1$ needs fewer atypical conditions to be present in order to generate the outcome $E S=1$. It requires only the instability of the executive, but not the presence of rum, other flammable materials, and so on. Hence, the revised account predicts that we are more strongly inclined to judge that $L L=1$ is an actual cause of $E S=1$ than $M=1$. Nonetheless, the witness for $M=1$ being a cause is still more normal than the actual world, so we still have some inclination to judge it an actual cause. As Moore [2009] recommends, the revised account yields a graded notion of actual causation. 
Note that the extent to which we have attenuation of actual causation over a causal chain is not just a function of spatiotemporal distance or the number of links. It is, rather, a function of how abnormal the circumstances are that must be in place if the causal chain is going to run from start to finish. In the postscript of [Lewis 1986], Lewis uses the phrase "sensitive causation" to describe cases of causation that depend on a complex configuration of background circumstances. For example, he describes a case where he writes a strong letter of recommendation for candidate $A$, thus earning him a job and displacing second-place candidate $B$, who then accepts a job at her second choice of institutions, displacing runner-up $C$, who then accepts a job at another university, where he meets his spouse, and they have a child, who later dies. While Lewis claims that his writing the letter is indeed a cause of the death, it is a highly sensitive cause, requiring an elaborate set of detailed conditions to be present. Woodward [2006] says that such causes are "unstable". Had the circumstances been slightly different, writing the letter would not have produced this effect (either the effect would not have occurred, or it would not have been counterfactually dependent on the letter). Woodward argues that considerations of stability often inform our causal judgments. Our definition allows us to take these considerations into account.

\subsection{Legal doctrines of intervening causes}

In the law, it is held that one is not causally responsible for some outcome when one's action led to that outcome only via the intervention of a later agent's deliberate action, or some very improbable event. For example, if Anne negligently spills gasoline, and Bob carelessly throws a cigarette in the spill, then Anne's action is a cause of the fire. But if Bob maliciously throws a cigarette in the gas, then Anne is not considered a cause [Hart and Honoré 1985].16 This reasoning often seems strange to philosophers, but legal theorists find it very natural. As we now show, we can model this judgment in our framework.

In order to fully capture the legal concepts, we need to represent the mental states of the agents. We can do this with the following six variables:

- $A N=1$ if Anne is negligent, 0 if she isn't;

- $A S=1$ if Anne spills the gasoline, 0 if she doesn't;

- $B C=1$ if Bob is careless (i.e. doesn't notice the gasoline), 0 if not;

- $B M=1$ if Bob is malicious, 0 otherwise;

- $B T=1$ if Bob throws a cigarette, 0 if he doesn't;

- $F=1$ if there is a fire, 0 if there isn't.

\footnotetext{
${ }^{16}$ This example is based on the facts of Watson v. Kentucky and Indiana Bridge and Railroad [1910].
} 
We have the following equations:

$$
\begin{aligned}
& F=\min (A S, B T) \\
& A S=A N \\
& B T=\max (B C, B M, 1-A S) .
\end{aligned}
$$

This model is shown graphically in Figure 4. Note that we have made somewhat arbitrary stipulations about what happens in the case where Bob is both malicious and careless, and in the cases where Anne does not spill; this is not clear from the usual description of the example. These stipulations do not affect our analysis.

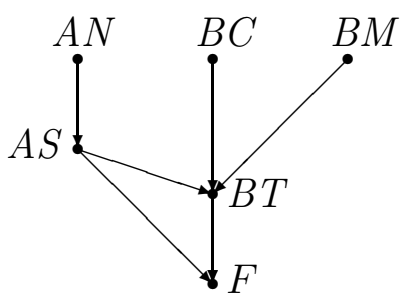

Figure 4: An intervening cause.

We assume that $B M, B C$, and $A N$ typically take the value 0 . But we can say more. In the law, responsibility requires a mens rea-literally a guilty mind. Mens rea comes in various degrees. Starting with an absence of mens rea, and then in ascending order or culpability, we have:

- prudent and reasonable-the defendant behaved as a reasonable person would;

- negligent-the defendant should have been aware of the risk of harm from his actions;

- reckless-the defendant acted in full knowledge of the harm her actions might cause;

- criminal/intentional-the defendant intended the harm that occurred.

In our framework, we can represent this scale by decreasing levels of typicality. While our practice so far has been to avoid comparisons of typicality between variables, such a comparison clearly seems warranted in this case. Specifically, we have in decreasing order of typicality:

1. $B C=1$;

2. $A N=1$;

3. $B M=1$. 
Thus, when we compare the normality of worlds in which two of these variables take the value 0 , and one takes the value 1, we would expect the world with $B C=1$ to be most normal, the world with $A N=1$ to be next most normal, and the world with $B M=1$ to be least normal. Consider first the case where Bob is careless. Then in the actual world we have

$$
(B M=0, B C=1, B T=1, A N=1, A S=1, F=1) .
$$

In our structural equations, $F=1$ depends counterfactually on both $B C=1$ and $A N=1$. Thus Definition 3.1 rules that both are actual causes. (We can just take $\vec{W}=\emptyset$ in both cases.) The best witness for $A N=1$ is

$$
(B M=0, B C=1, B T=1, A N=0, A S=0, F=0),
$$

while the best witness for $B C=1$ is

$$
(B M=0, B C=0, B T=0, A N=1, A S=1, F=0) .
$$

Both of these worlds are more normal than the actual world. The first is more normal because $A N$ takes the value 0 instead of 1 . The second world is more normal than the actual world because $B C$ takes the value 0 instead of 1 . Hence, the revised theory judges that both are actual causes. However, the best witness for $A N=1$ is more normal than the best witness for $B C=1$. The former witness has $B C=1$ and $A N=0$, while the latter witness has $B C=0$ and $A N=1$. Since $A N=1$ is more atypical than $B C=1$, the first witness is more normal. This means that we are more inclined to judge that Anne's negligence is an actual cause of the fire than that Bob's carelessness is.

Now consider the case where Bob is malicious. The actual world is

$$
(B M=1, B C=0, B T=1, A N=1, A S=1, F=1) .
$$

Again, Definition 3.1 straightforwardly rules that both $B M=1$ and $A N=1$ are actual causes. The best witness for $A N=1$ is

$$
(B M=1, B C=0, B T=1, A N=0, A S=0, F=0),
$$

while the best witness for $B M=1$ is

$$
(B M=0, B C=0, B T=0, A N=1, A S=1, F=0) .
$$

Again, both of these worlds are more normal than the actual world, so our revised theory judges that both are actual causes. However, now the best witness for $A N=1$ is less normal than the best witness for $B M=1$, since $B M=1$ is more atypical than $A N=1$. So now our theory predicts that we are more inclined to judge that Bob's malice is an actual cause of the fire than that Anne's negligence is.

Recall that in Section 7.2 we saw how judgments of the causal status of the administrative assistant's action changed, depending on the normative status of the professor's action. Something similar is happening here: the causal status of Anne's action changes with the normative status of Bob's action. This example also illustrates how context can play a role in determining what is normal and abnormal. In the legal context, there is a clear ranking of norm violations. 


\subsection{Preemption and short circuits}

Recall our example of preemption (Example 3.3). in which an assassin poisoned the victim's drink with a backup present. It is convenient to start our exposition with a simplified causal model that omits the variable representing the backup's readiness. (Recall that for purposes of showing the assassin's action to be an actual cause, it did not much matter what we did with that variable.) Thus our variables are:

- $A=1$ if the assassin poisons the drink, 0 if not;

- $B=1$ if the backup poisons the drink, 0 if not;

- $D=1$ if the victim dies, 0 if not.

The equations are

$$
\begin{aligned}
& B=(1-A) ; \\
& D=\max (A, B) .
\end{aligned}
$$

The structure is the same as that depicted in Figure 3, with $R$ omitted. In the actual world, $A=1, B=0, D=1$. Definition 3.1 rules that $A=1$ is an actual cause of $D=1$. We can let $\vec{W}=\{B\}$, and $\vec{w}=\{0\}$. This satisfies clause 2 of Definition 3.1. We thus get that the world ( $A=0, B=0, D=0)$ is a witness for $A=1$ being an actual cause of $D=1$.

On the new account, however, this is not enough. We must also ensure that the witness is sufficiently normal. It seems natural to take $A=0$ and $B=0$ to be typical, and $A=1$ and $B=1$ to be atypical. It is morally wrong, unlawful, and highly unusual for an assassin to be poisoning one's drink. This gives rise to a normality ranking in which the witness $(A=$ $0, B=0, D=0)$ is more normal than the actual world in which $(A=1, B=0, D=1)$. So the revised account still rules that $A=1$ is an actual cause of $D=1$.

Now let us reconsider Example 4.2, in which two bodyguards conspire to make it appear as if one of them has foiled an assassination attempt. Let us model this story using the following variables:

- $A=1$ if the first bodyguard puts in the antidote, 0 otherwise;

- $P=1$ if the second bodyguard puts in the poison, 0 otherwise;

- $V S=1$ if the victim survives, 0 otherwise.

The equations are

$$
\begin{aligned}
& P=A \\
& V S=\max (A,(1-P)) .
\end{aligned}
$$

In the actual world, $A=1, P=1$, and $V S=1$. This model is shown graphically in Figure 5. A quick inspection reveals that this model is isomorphic to the model for our preemption case, substituting $P$ for $1-B$ and $V S$ for $D$. And indeed, Definition 3.1 rules that 
$A=1$ is an actual cause of $V S=1$, with $(A=0, P=1, V S=0)$ as witness. Intuitively, if we hold fixed that the second bodyguard added the poison, the victim would not have survived if the first bodyguard had not added the antidote first. Yet intuitively, many people judge that the first bodyguard's adding the antidote is not a cause of survival (or a preventer of death), since the only threat to victim's life was itself caused by that very same action.

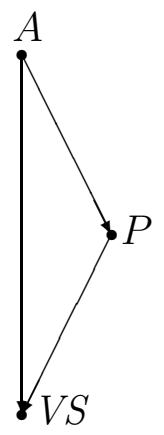

Figure 5: The poisoning example reconsidered.

In order to capture the judgment that $A=1$ is not an actual cause of $V S=1$, we must appeal to the normality ranking. Here we have to be careful. Suppose that we decide that the typical value of both $A$ and $P$ is 0 , and the atypical value 1 . This would seem prima facie reasonable. This would give us a normality ordering in which the witness $(A=0, P=$ $1, V S=0)$ is more normal than the actual world $(A=1, P=1, V S=1)$. Intuitively, the value of $A$ is more typical in the first world, and the value of $P$ is the same in both words, so the first world is more normal overall than the second. If we reason this way, the modified theory still makes $A=1$ an actual cause of $V S=1$.

There is, however, a subtle mistake in this way of thinking about the normality ordering. The variable $P$ is not fixed by exogenous variables that are outside of the model; it is also determined by $A$, according to the equation $P=A$. This means that when we think about what is typical for $P$, we should rank not just the typicality of particular values of $P$, but the typicality of different ways for $P$ to depend on $A$. And the most natural ranking is the following, in decreasing order of typicality:

1. $P=0$, regardless of $A$;

2. $P=A$;

3. $P=1$ regardless of $A$.

That is, the most typical situation is one where there is no possibility of poison going into the drink. The next most typical is the one that actually occurred. Least typical is one where the second bodyguard puts in poison even when no antidote is added. Put another way, the most typical situation is one where the second bodyguard has no bad intentions, next most typical is 
where he has deceitful intentions, and least typical is where he has homicidal intentions. This is similar to the ranking we saw in the previous example. Therefore, in the witness world, where $A=0$ and $P=1, P$ depends on $A$ in a less typical way than in the actual world, in which $A=1$ and $P=1$. On this interpretation, the witness world $(A=0, P=1, V S=0)$ is no longer more normal than the actual world $(A=1, P=1, V S=0)$. In fact, using the guidelines we have been following, these two worlds are incomparable.

Thus, while the pattern of counterfactual dependence is isomorphic in the two cases, the normality ranking on worlds is not. The result is that in the case of simple preemption, the witness is more normal than the actual world, while in the second case it is not. This explains the difference in our judgments of actual causation in the two examples. 17

This example raises an interesting technical point. Our treatment of this example involves an exception to the default assumption that it is typical for endogenous variables to depend on one another in accordance with the structural equations. (Observant readers will note that our treatment of the preemption example earlier in this section did as well. 18 We can avoid this by adding an additional variable to the model, akin to the variable representing the backup's readiness in our discussion of the preemption example (Example 3.3). Let $I$ represent the intentions of the second bodyguard, as follows:

- $I=0$ if he has benign intentions;

- $I=1$ if he has deceitful intentions;

- $I=2$ if he has murderous intentions.

Now we can rewrite the equations as follows:

$$
\begin{aligned}
& P=f(A, I) ; \\
& V S=\max (A,(1-P)),
\end{aligned}
$$

where $f(A, I)$ is the function of $A$ and $I$ that takes the value $0, A$, or 1 when $I=0$, 1 , or 2 , respectively. This model is shown graphically in Figure 6, In this model, we can retain our guideline that endogenous variables typically obey the structural equations. That is, the typicality or atypicality of the way in which $P$ depends on $A$ can be coded by the typicality or atypicality of the values of $I$. Assume that atypicality increases with increasing values of $I$. Now the best witnesses for $A=1$ being an actual cause of $V S=1$ are $(A=0, I=0, P=$

\footnotetext{
${ }^{17}$ Hall [2007, Sections 3.3 and 3.4] presents several examples of what he calls "short circuits". These can be handled in essentially the same way.

${ }^{18} \mathrm{~A}$ similar strategy might be used to address an example proposed by an anonymous referee. Suppose there are abnormally heavy rains. Nonetheless, a dam holds, and a town is spared from a flood. If the dam's remaining intact is typical, and the dam breaking is atypical, then it appears that our account must rule that the dam's holding is not an actual cause of the town's being spared. However, we could have the typicality of a dam break depend upon the heavy rain. Even though the dam does not in fact break under heavy rains, it would not be atypical for the dam to break under such conditions. That is, even though the actual behavior of the dam does not depend upon the rain, its typical behavior does.
} 
$1, V S=0)$ and $(A=0, I=2, P=1, V S=0)$. The first of these involves $P$ behaving less typically than in the actual world (since it now violates the equation $P=f(A, I)$ ). The second witness involves $I$ taking a less typical value ( 2 instead of 1$)$. In the second witness, $P$ is behaving typically: it satisfies the equation $P=f(A, I)$. The abnormality of the situation is now attributed to $I$. Neither of these witnesses would be as normal as the actual world, so our revised theory would still rule that $A=1$ is not an actual cause of $V S=1$.

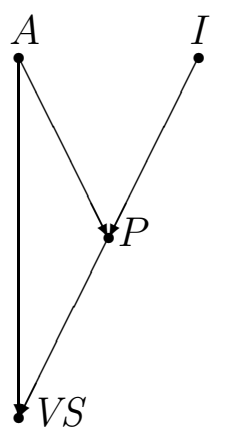

Figure 6: The poisoning example, with intentions.

This raises an interesting technical question. Is it always possible to perform this kind of maneuver? That is, if we have a theory of typicality in which one variable typically violates one of the structural equations, is it always possible, through the addition of suitable variables, to reproduce the normality ordering on worlds using a theory of typicality in which the structural equations are typically obeyed? We conjecture that it is always possible to formally construct additional variables to do this; but there is no guarantee that such variables will correspond to any causal variables that are actually present in a particular situation.

\section{Conclusion}

We have shown how considerations of normality can be added to a theory of causality based on structural equations, and have examined the advantages of doing so. Doing this gives us a convenient way of dealing with two problems that we have called the problem of isomorphism and the problem of disagreement. While we have done our analysis in the context of the HP definition of causality, the ideas should carry over to other definitions based on structural equations. We hope that future empirical work will examine more carefully how people use normality in judgments of actual causation, and that future philosophical work will examine more carefully the reasons why this might be desirable.

\section{References}

Adams, E. (1975). The Logic of Conditionals. Dordrecht, Netherlands: Reidel. 
Alicke, M. (1992). Culpable causation. Journal of Personality and Social Psychology 63, 368-378.

Alicke, M. D., D. Rose, and D. Bloom (2011). Causation, norm violation, and culpable control. Journal of Philosophy 108, 670-696.

Beebee, N. (2004). Causing and nothingness. In J. Collins, N. Hall, and L. A. Paul (Eds.), Causation and Counterfactuals, pp. 291-308. Cambridge, Mass.: MIT Press.

Chockler, H. and J. Y. Halpern (2004). Responsibility and blame: A structural-model approach. Journal of A.I. Research 20, 93-115.

Cushman, F., J. Knobe, and W. Sinnott-Armstrong (2008). Moral appraisals affect doing/allowing judgments. Cognition 108(1), 281-289.

Danks, D. (2013). Functions and cognitive bases for the concept of actual causation. Erkenntnis. To appear.

Dowe, P. (2000). Physical Causation. Cambridge, U.K.: Cambridge University Press.

Dubois, D. and H. Prade (1991). Possibilistic logic, preferential models, non-monotonicity and related issues. In Proc. Twelfth International Joint Conference on Artificial Intelligence (IJCAI'91), pp. 419-424.

Geffner, H. (1992). High probabilities, model preference and default arguments. Mind and Machines 2, 51-70.

Glymour, C., D. Danks, B. Glymour, F. Eberhardt, J. Ramsey, R. Scheines, P. Spirtes, C. M. Teng, and J. Zhang (2010). Actual causation: a stone soup essay. Synthese 175, 169-192.

Glymour, C. and F. Wimberly (2007). Actual causes and thought experiments. In J. Campbell, M. O'Rourke, and H. Silverstein (Eds.), Causation and Explanation, pp. 43-67. Cambridge, MA: MIT Press.

Goldszmidt, M. and J. Pearl (1992). Rank-based systems: a simple approach to belief revision, belief update and reasoning about evidence and actions. In Principles of Knowledge Representation and Reasoning: Proc. Third International Conference (KR '92), pp. 661-672.

Hall, N. (2004). Two concepts of causation. In J. Collins, N. Hall, and L. A. Paul (Eds.), Causation and Counterfactuals. Cambridge, Mass.: MIT Press.

Hall, N. (2007). Structural equations and causation. Philosophical Studies 132, 109-136.

Halpern, J. Y. (2008). Defaults and normality in causal structures. In Principles of Knowledge Representation and Reasoning: Proc. Eleventh International Conference (KR '08), pp. 198-208.

Halpern, J. Y. and C. Hitchcock (2010). Actual causation and the art of modeling. In Causality, Probability, and Heuristics: A Tribute to Judea Pearl, pp. 383-406. London: College Publications. 
Halpern, J. Y. and C. Hitchcock (2012). Compact representations of causal models. Unpublished manuscript.

Halpern, J. Y. and J. Pearl (2001). Causes and explanations: A structural-model approach. Part I: Causes. In Proc. Seventeenth Conference on Uncertainty in Artificial Intelligence (UAI 2001), pp. 194-202.

Halpern, J. Y. and J. Pearl (2005). Causes and explanations: A structural-model approach. Part I: Causes. British Journal for Philosophy of Science 56(4), 843-887.

Hart, H. L. A. and T. Honoré (1985). Causation in the Law (second ed.). Oxford, U.K.: Oxford University Press.

Hiddleston, E. (2005). Causal powers. British Journal for Philosophy of Science 56, 27-59.

Hitchcock, C. (2001). The intransitivity of causation revealed in equations and graphs. Journal of Philosophy XCVIII(6), 273-299.

Hitchcock, C. (2007). Prevention, preemption, and the principle of sufficient reason. Philosophical Review 116, 495-532.

Hitchcock, C. (2012). Portable causal dependence: a tale of consilience. Philosophy of Science 79, 942-951.

Hitchcock, C. and J. Knobe (2009). Cause and norm. Journal of Philosophy 106, 587-612.

Huber, F. (2011). Structural equations and beyond. Unpublished manuscript.

Hume, D. (1748). An Enquiry Concerning Human Understanding. Reprinted by Open Court Press, LaSalle, IL, 1958.

Kahneman, D. and D. T. Miller (1986). Norm theory: comparing reality to its alternatives. Psychological Review 94(2), 136-153.

Kahneman, D. and A. Tversky (1982). The simulation heuristic. In D. Kahneman, P. Slovic, and A. Tversky (Eds.), Judgment Under Incertainty: Heuristics and Biases, pp. 201210. Cambridge/New York: Cambridge University Press.

Knobe, J. and B. Fraser (2008). Causal judgment and moral judgment: two experiments. In W. Sinnott-Armstrong (Ed.), Moral Psychology, Volume 2: The Cognitive Science of Morality, pp. 441-447. Cambridge, MA: MIT Press.

Kraus, S., D. Lehmann, and M. Magidor (1990). Nonmonotonic reasoning, preferential models and cumulative logics. Artificial Intelligence 44, 167-207.

Lewis, D. (1973). Causation. Journal of Philosophy 70, 556-567. Reprinted with added "Postscripts" in D. Lewis, Philosophical Papers, Volume II, Oxford University Press, 1986, pp. 159-213.

Lewis, D. (1979). Counterfactual dependence and time's arrow. Noûs 13, 455-476. Reprinted in D. Lewis, Philosophical Papers, Volume II, Oxford University Press, 1986, pp. 32-52. 
Lewis, D. (1986). Causation. In Philosophical Papers, Volume II, pp. 159-213. New York: Oxford University Press. The original version of this paper, without numerous postscripts, appeared in the Journal of Philosophy 70, 1973, pp. 113-126.

Lewis, D. (2000). Causation as influence. Journal of Philosophy XCVII(4), 182-197.

Lewis, D. (2004). Void and object. In J. Collins, N. Hall, and L. A. Paul (Eds.), Causation and Counterfactuals, pp. 277-290. Cambridge, Mass.: MIT Press.

Livengood, J. (2013). Actual causation in simple voting scenarios. Nous. To appear.

Lombrozo, T. (2010). Causal-explanatory pluralism: how intentions, functions, and mechanisms influence causal ascriptions. Cognitive Psychology 61(4), 303-332.

Marek, W. and M. Truszczyński (1993). Nonmonotonic Logic. Berlin/New York: SpringerVerlag.

Maudlin, T. (2004). Causation, counterfactuals, and the third factor. In J. Collins, N. Hall, and L. A. Paul (Eds.), Causation and Counterfactuals, pp. 419-444. Cambridge, Mass.: MIT Press.

McGrath, S. (2005). Causation by omission. Philosophical Studies 123, 125-148.

Menzies, P. (2004). Causal models, token causation, and processes. Philosophy of Science $71,820-832$.

Menzies, P. (2007). Causation in context. In H. Price and R. Corry (Eds.), Causation, Physics, and the Constitution of Reality, pp. 191-223. Oxford, U.K.: Oxford University Press.

Mill, J. S. (1856). A System of Logic, Ratiocinative and Inductive (Fourth ed.). London: John W. Parker and Son.

Moore, M. S. (2009). Causation and Responsibility. Oxford, UK: Oxford University Press.

Pearl, J. (1989). Probabilistic semantics for nonmonotonic reasoning: a survey. In Proc. First International Conference on Principles of Knowledge Representation and Reasoning (KR '89), pp. 505-516. Reprinted in G. Shafer and J. Pearl (Eds.), Readings in Uncertain Reasoning, pp. 699-710. San Francisco: Morgan Kaufmann, 1990.

Pearl, J. (2000). Causality: Models, Reasoning, and Inference. New York: Cambridge University Press.

Regina v. Faulkner (1877). Court of Crown Cases Reserved, Ireland). In 13 Cox Criminal Cases 550.

Reiter, R. (1980). A logic for default reasoning. Artificial Intelligence 13, 81-132.

Reiter, R. (1987). Nonmonotonic reasoning. In J. F. Traub, B. J. Grosz, B. W. Lampson, and N. J. Nilsson (Eds.), Annual Review of Computer Science, Volume 2, pp. 147-186. Palo Alto, Calif.: Annual Reviews Inc.

Roxborough, C. and J. Cumby (2009). Folk psychological concepts: causation. Philosophical Psychology 22, 205-213. 
Schaffer, J. (2000). Causation by disconnection. Philosophy of Science 67, 285-300.

Schaffer, J. (2004). Causes need not be physically connected to their effects. In C. Hitchcock (Ed.), Contemporary Debates in Philosophy of Science, pp. 197-216. Oxford, U.K.: Basil Blackwell.

Shoham, Y. (1987). A semantical approach to nonmonotonic logics. In Proc. 2nd IEEE Symposium on Logic in Computer Science, pp. 275-279. Reprinted in M. L. Ginsberg (Ed.), Readings in Nonmonotonic Reasoning, pp. 227-250. San Francisco: Morgan Kaufman, 1987.

Spohn, W. (1988). Ordinal conditional functions: a dynamic theory of epistemic states. In W. Harper and B. Skyrms (Eds.), Causation in Decision, Belief Change, and Statistics, Volume 2, pp. 105-134. Dordrecht, Netherlands: Reidel.

Sytsma, J., J. Livengood, and D. Rose (2010). Two types of typicality: Rethinking the role of statistical typicality in ordinary causal attributions. Studies in History and Philosophy of Biological and Biomedical Sciences 43, 814-820.

Tversky, A. and D. Kahneman (1973). Availability: a heuristic for judging frequency and probability. Cognitive Psychology 5, 207-232.

Watson v. Kentucky and Indiana Bridge and Railroad (1910). 137 Kentucky 619. In $126 \mathrm{SW}$ 146.

Weslake, B. (2011). A partial theory of actual causation. Unpublished manuscript.

Woodward, J. (2003). Making Things Happen: A Theory of Causal Explanation. Oxford, U.K.: Oxford University Press.

Woodward, J. (2006). Sensitive and insensitive causation. Philosophical Review 115, 1-50.

Wright, R. W. (1985). Causation in tort law. California Law Review 73, 1735-1828. 